\title{
Anisotropic magnetoresistance of spin-orbit coupled carriers scattered from polarized magnetic impurities
}

\author{
Maxim Trushin, ${ }^{1,2}$ Karel Výborný, ${ }^{3}$ Peter Moraczewski, ${ }^{4}$ Alexey A. Kovalev, ${ }^{5}$ John Schliemann, ${ }^{1}$ and T. Jungwirth ${ }^{3,6}$ \\ ${ }^{1}$ Institut für Theoretische Physik, Universität Regensburg, D-93040 Regensburg, Germany \\ ${ }^{2}$ Physics Department, University of Texas, 1 University Station C1600, Austin, Texas 78712, USA \\ ${ }^{3}$ Institute of Physics, Academy of Sciences of the Czech Republic, v.v.i., Cukrovarnická 10, Praha 6 CZ-16253, Czech Republic \\ ${ }^{4}$ I. Institut für Theoretische Physik, Universität Hamburg, Jungiusstrasse 9, 20355 Hamburg, Germany \\ ${ }^{5}$ Department of Physics and Astronomy, University of California, Los Angeles, California 90095, USA \\ ${ }^{6}$ School of Physics and Astronomy, University of Nottingham, Nottingham NG7 2RD, United Kingdom
}

(Received 24 April 2009; revised manuscript received 7 September 2009; published 7 October 2009)

\begin{abstract}
Anisotropic magnetoresistance (AMR) is a relativistic magnetotransport phenomenon arising from combined effects of spin-orbit coupling and broken symmetry of a ferromagnetically ordered state of the system. In this work we focus on one realization of the AMR in which spin-orbit coupling enters via specific spin-textures on the carrier Fermi surfaces and ferromagnetism via elastic scattering of carriers from polarized magnetic impurities. We report detailed heuristic examination, using model spin-orbit coupled systems, of the emergence of positive AMR (maximum resistivity for magnetization along current), negative AMR (minimum resistivity for magnetization along current), and of the crystalline AMR (resistivity depends on the absolute orientation of the magnetization and current vectors with respect to the crystal axes) components. We emphasize potential qualitative differences between pure magnetic and combined electromagnetic impurity potentials, between short-range and long-range impurities, and between spin-1/2 and higher spin-state carriers. Conclusions based on our heuristic analysis are supported by exact solutions to the integral form of the Boltzmann transport equation in archetypical two-dimensional electron systems with Rashba and Dresselhaus spin-orbit interactions and in the three-dimensional spherical Kohn-Littinger model. We include comments on the relation of our microscopic calculations to standard phenomenology of the full angular dependence of the AMR, and on the relevance of our study to realistic, two-dimensional conduction-band carrier systems and to anisotropic transport in the valence band of diluted magnetic semiconductors.
\end{abstract}

DOI: 10.1103/PhysRevB.80.134405

PACS number(s): 72.10.-d, 72.20.My

\section{INTRODUCTION}

Advanced theoretical approaches and experiments in new unconventional ferromagnets have recently led to a renewed interest in the relativistic, extraordinary magnetotransport effects. There are two distinct extraordinary magnetoresistance coefficients, the anomalous Hall effect (AHE) and the anisotropic magnetoresistance (AMR). The AHE is the antisymmetric transverse magnetoresistance coefficient obeying $\rho_{x y}(\mathbf{M})=-\rho_{x y}(-\mathbf{M})$, where the magnetization vector $\mathbf{M}$ is pointing perpendicular to the $\hat{x}, \hat{y}$ plane of a Hall bar sample. The AMR is the symmetric coefficient with the longitudinal and transverse resistivities obeying, $\rho_{x x}(\mathbf{M})=\rho_{x x}(-\mathbf{M})$ and $\rho_{x y}(\mathbf{M})=\rho_{x y}(-\mathbf{M})$, where $\mathbf{M}$ has an arbitrary orientation but in most studies it lies in the $x-y$ plane. Numerous works have explored the origins of the AHE; for reviews see, e.g., Refs. 1-3. Diluted magnetic semiconductors became one of the favorable test bed systems for AHE investigation ${ }^{4-10}$ due to their tunability and the relatively simple, yet strongly spinorbit coupled Fermi surfaces. ${ }^{11,12}$ An even more systematic and comprehensive understanding of the AHE on a model level has been obtained by considering two-dimensional semiconductor systems with archetypical spin-orbit interactions (SOIs) of the Rashba and Dresselhaus type. ${ }^{3,13-23}$

Despite the long history and importance in magnetic recording technologies, the AMR has been studied less extensively. ${ }^{24-28}$ Similar to the AHE, it has been recently argued that the analysis of the AMR can be significantly simplified in diluted magnetic semiconductors such as (Ga,Mn)As. ${ }^{29,30}$ Two distinct microscopic mechanisms have been identified that can lead to anisotropic carrier lifetimes in these systems: One combines the spin-orbit coupling in an unpolarized carrier band with scattering off polarized magnetic impurities while the other emphasizes polarization of the carrier band itself and does not require magnetic nature of the scatterers. (Note that apart from lifetimes, the AMR may also arise from anisotropic group velocities. ${ }^{49}$ ) Although acting simultaneously in real systems, theoretically both mechanisms can be turned on and off independently and it was found ${ }^{29}$ that the scattering of spin-orbit coupled band carriers from magnetically polarized impurities should dominate in the diluted magnetic semiconductors. Building on the analogy with AHE studies we seek further insight into the basic physics of this AMR mechanism by focusing on the archetypical spin-orbit coupled two-dimensional systems.

Using the relaxation-time approximation (RTA) and starting with the Rashba and Dresselhaus models we show in Sec. II how the sign of the AMR can be inferred by inspecting the spin texture of the spin-orbit coupled Fermi surface. We point out that impurities containing polarized magnetic potential only or containing a combined electromagnetic potential can yield distinct AMR phenomenologies. Examination of the Rashba and Dresselhaus models allows us to draw separate links between the spin-texture and the noncrystalline and crystalline AMR components where the noncrystalline AMR depends on the relative angle between $\mathbf{M}$ and 
current I while the crystalline AMR has an additional dependence on the absolute orientation of $\mathbf{M}$ and $\mathbf{I}$ in the coordinate system of the crystal axes. We conclude the qualitative discussion in Sec. II by illustrating in the RashbaDresselhaus system a potentially important effect on AMR of long-range impurities, and in a spherical Kohn-Luttinger model $^{29}$ the effect of carriers with higher spin state. Analysis of these effects relates our work to previous theoretical studies of the AMR in $(\mathrm{Ga}, \mathrm{Mn}) \mathrm{As}$ diluted magnetic semiconductors. ${ }^{29,31,37,38}$ The validity of the heuristic analysis of the AMR is confirmed in Sec. III where we explain the relation between the RTA and the exact solution to the integral Boltzmann equation. ${ }^{32}$ Quantitative results for the AMR are derived in this section and Appendix for the Rashba model and for the Dresselhaus model with short-range electromagnetic impurities and for the combined RashbaDresselhaus model with arbitrary strength of the two SOI terms and with short-range magnetic impurities. In Sec. IV we comment on the relevance of our model calculations to realistic two-dimensional semiconductor structures.

\section{HEURISTIC LINK BETWEEN SPIN TEXTURES AND IMPURITY POTENTIALS AND THE AMR}

We limit our discussion in this section to AMRs defined as the relative difference between longitudinal resistivities for magnetization aligned parallel and perpendicular to the current direction. In situations discussed below, the transverse resistivity vanishes and we can define

$$
\operatorname{AMR}=\frac{\rho_{\mathbf{I}}^{\|}-\rho_{\hat{\mathbf{I}}}^{\perp}}{\left(\rho_{\hat{\mathbf{I}}}^{\|}+\rho_{\hat{\mathbf{I}}}^{\perp}\right) / 2}=\frac{\sigma_{\hat{\mathbf{I}}}^{\perp}-\sigma_{\hat{\mathbf{I}}}^{\|}}{\left(\sigma_{\hat{\mathbf{I}}}^{\|}+\sigma_{\hat{\mathbf{I}}}^{\perp}\right) / 2},
$$

where $\rho_{\hat{\mathbf{I}}}^{\|}\left(\sigma_{\hat{\mathbf{I}}}^{\|}\right)$and $\rho_{\hat{\mathbf{I}}}^{\perp}\left(\sigma_{\hat{\mathbf{I}}}^{\perp}\right)$ is the longitudinal resistivity (conductivity) for $\mathbf{M} \| \mathbf{I}$ and for $\mathbf{M} \perp \mathbf{I}$, respectively, and the subscript $\hat{\mathbf{I}}$ labels the orientation of current with respect to crystal axes. (The relation of our microscopic theory to the standard phenomenology of the full angular dependence of the AMR will be commented upon in Sec. III.) Our heuristic analysis of the AMR defined in Eq. (1) is based on the RTA and on assuming a proportionality between resistivity and the first-order Born approximation to elastic scattering probabilities from the state with the group velocity along I. Furthermore we consider only the strongest contribution to the transport lifetime which comes from backscattering, i.e., from transitions into states with group velocity opposite to $\mathbf{I}$. We use these approximations and consider several archetypical spin-orbit coupled Fermi surfaces to elucidate the relation of the spin texture and nature of the impurity potential to various fundamental aspects of the AMR phenomenology.

\section{A. AMR in the Rashba model}

We start with the two-dimensional electron system with Rashba SOI which yields positive AMR independent of the current orientation in the crystal, and demonstrate the potential qualitative difference between pure magnetic short-range impurity potential $\propto \hat{\mathbf{e}}_{M} \cdot \hat{\mathbf{s}} / s$ and a combined electromagnetic
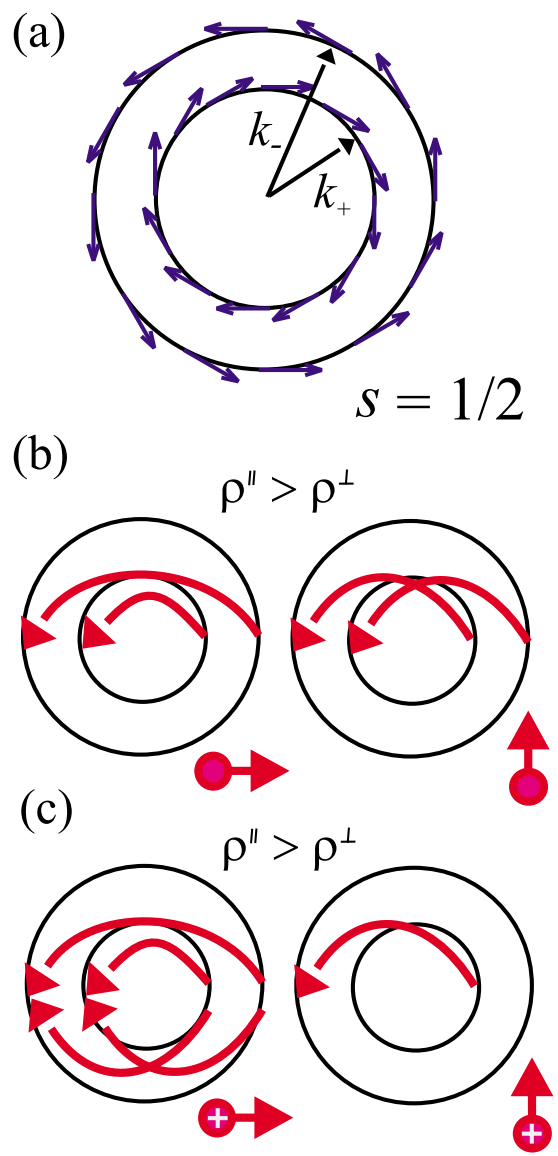

FIG. 1. (Color online) Rashba model and (a) its spin texture along the Fermi contours. Dominant scattering channels for the states with group velocity pointing to the right when (b) magnetic and (c) electromagnetic impurities (see text) constitute the prevalent source of momentum relaxation. Note the indicated directions of impurity polarization. The current flow is directed from left to right. The reader might consider the limit $k_{-} \gg k_{+}$for better understanding of this and subsequent figures.

potential $\propto 1+\hat{\mathbf{e}}_{M} \cdot \hat{\mathbf{s}} / s$. Here $\hat{\mathbf{e}}_{M}$ denotes the magnetization unit vector and $\hat{\mathbf{s}}$ is the carrier spin operator. For electrons with $s=1 / 2$, the operator $\hat{\mathbf{s}} / s$ can be represented by the 2 $\times 2$ Pauli matrices $\boldsymbol{\sigma}=\left(\sigma_{x}, \sigma_{y}, \sigma_{z}\right)$.

The tangential spin-texture along the Fermi contour of the Rashba Hamiltonian,

$$
H_{R}=\frac{\hbar^{2} k^{2}}{2 m}+\alpha\left(\sigma_{x} k_{y}-\sigma_{y} k_{x}\right),
$$

is shown in Fig. 1(a). The spinors on the majority (-) and minority (+) Rashba band are given by $\left|\mathbf{k}_{ \pm}\right\rangle=\left(1, \mp i e^{i \theta}\right)$, where $\tan \theta=k_{y} / k_{x}$. From now on the coordinate system is chosen in such a way that $\hat{x}, \hat{y}$, and $\hat{z}$ directions coincide with [100], [010], and [001] crystallographic axes, respectively, as shown in Fig. 2. Assuming current along $\hat{x}$ direction, we can infer the backscattering amplitudes of the states with the group velocity (k-vector) parallel to the current by recalling the following properties of the scattering matrix elements: 

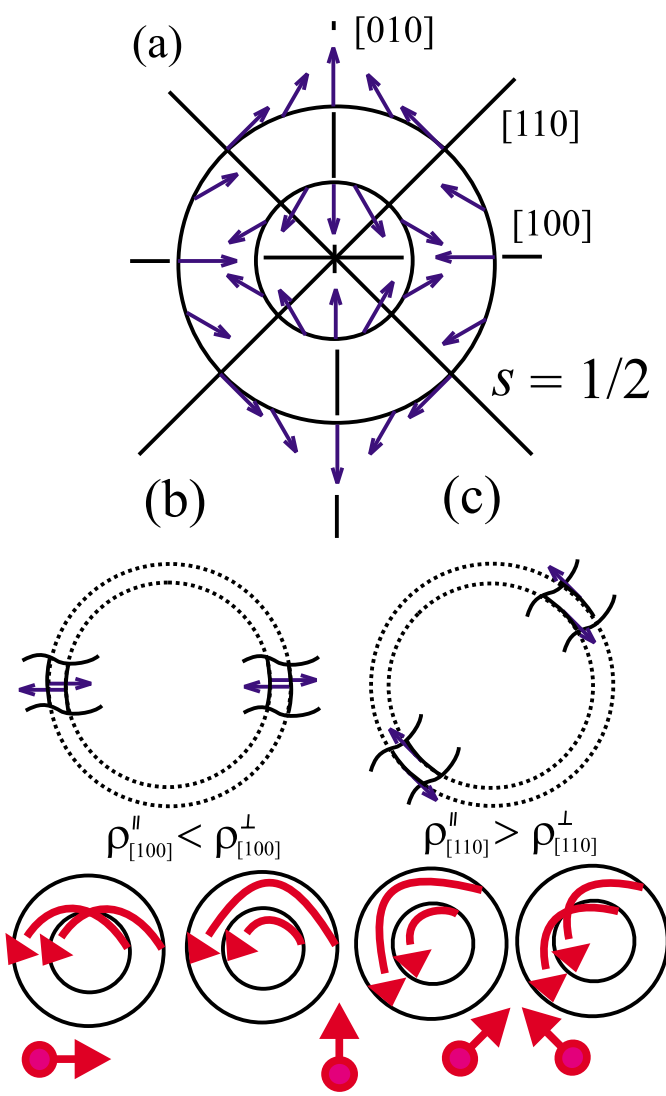

FIG. 2. (Color online) Dresselhaus model and (a) its spin texture. In order to determine the current and the AMR along the [100] and [110] crystallographic directions we focus on the states with group velocities pointing in the respective directions, (b) and (c). Dominant momentum relaxation channels for these states and scattering on magnetic impurities are indicated on the bottom panels.

$$
\begin{array}{ll}
\left\langle\downarrow\left|\sigma_{x}\right| \downarrow\right\rangle=0 \quad & \left\langle\uparrow\left|\sigma_{x}\right| \downarrow\right\rangle=1, \\
\left\langle\downarrow\left|\sigma_{y}\right| \downarrow\right\rangle=1 \quad & \left\langle\uparrow\left|\sigma_{y}\right| \downarrow\right\rangle=0 .
\end{array}
$$

Here we labeled the spinors by arrows whose orientation can be directly compared to the spin textures depicted in Fig. 1(a). The allowed backscattering processes, according to the relations in Eq. (3), are highlighted in Fig. 1(b) for the pure magnetic impurity potential. When magnetization points along the $\hat{x}$ direction [i.e., to the right in Fig. 1(b)], $\hat{e}_{M} \cdot \boldsymbol{\sigma}$ $=\sigma_{x}$ and the backscattering of states moving along the $\hat{x}$ direction is due to majority-to-majority and minority-tominority band transitions. In the case of magnetization parallel to the $\hat{y}$-direction, $\hat{e}_{M} \cdot \boldsymbol{\sigma}=\sigma_{y}$ and backscattering is due to majority-to-minority and minority-to-majority transitions. In the limit of $k_{-} \gg k_{+}$, these figures suggest that backscattering is strongly suppressed for $\mathbf{M} \perp \mathbf{I}$ implying low resistivity in this configuration compared to the $\mathbf{M} \| \mathbf{I}$ case. The AMR defined in Eq. (1) is therefore expected to have positive sign in the Rashba model. Quantitative Boltzmann equation calculations presented in Sec. III confirm the positive AMR for all $k_{-}>k_{+}$. They also confirm the vanishing magnitude of the AMR in the weak SOI, large Fermi energy limit $\left(k_{+} \approx k_{-}\right)$ which is discerned directly from our pictorial representation of the allowed backscattering transitions considering nearly degenerate majority and minority Rashba bands in Fig. 1(b).

The behavior of AMR in the limit of degenerate Rashba bands, while keeping the tangential spin textures, is qualitatively altered when the impurity potential contains magnetic and nonmagnetic components (e.g., for $\mathrm{Mn}$ acceptors in III-V semiconductors). Replacing $\sigma_{x, y}$ with $1+\sigma_{x, y}$ in the relations (3) allows us to illustrate this by again considering the transitions that contribute to the backscattering; note that this does not describe the situation where there are two distinct types of impurities ${ }^{50}$ (such as phonons and chargeneutral magnetic impurities). As highlighted in Fig. 1(c), there is now always one of the Rashba bands in which backscattering is absent for $\mathbf{M} \perp \mathbf{I}$, independent of the difference between $k_{+}$and $k_{-}$. For $\mathbf{M} \| \mathbf{I}$, backscattering occurs in both bands and each of the states moving along the current can scatter to both majority and minority band states. This implies large positive AMR even in the limit of $k_{+} \approx k_{-}$.

Finally we point out that the circular symmetry of the Rashba spin-texture makes the model a prototype realization of a purely noncrystalline AMR system. The AMR is independent of the orientation of current in the coordinate system of crystallographic axes and depends only on the relative angle between $\mathbf{M}$ and $\mathbf{I}$.

\section{B. AMR in the Dresselhaus model}

The tangential spin-1/2 texture of the Rashba model represents arguably the simplest host for a positive purely noncrystalline AMR. The Dresselhaus SOI can be viewed as a minimal model demonstrating the link between a radial spin$1 / 2$ texture and a negative AMR, and illustrating the emergence of crystalline AMR. The Dresselhaus Hamiltonian,

$$
H_{D}=\frac{\hbar^{2} k^{2}}{2 m}+\beta\left(\sigma_{x} k_{x}-\sigma_{y} k_{y}\right),
$$

yields the majority and minority eigenstates, $\left|\mathbf{k}_{ \pm}\right\rangle$ $=\left(1, \pm e^{-i \theta}\right)$, whose spin orientations along the respective Fermi contours are depicted in Fig. 2(a). We can use the same analysis of the backscattering amplitudes as in the previous subsection to link this spin texture to the expected basic AMR phenomenology in the Dresselhaus model.

In Fig. 2(b), we consider the case of current flowing along the $\hat{x}$ direction ([100] crystal axis) and scattering from impurities carrying the short-range magnetic potential only. Using the same representation of the spinors as in Eqs. (3) we can write

$$
\begin{array}{ll}
\left\langle\rightarrow\left|\sigma_{x}\right| \rightarrow\right\rangle=1 \quad\left\langle\leftarrow\left|\sigma_{x}\right| \rightarrow\right\rangle=0, \\
\left\langle\rightarrow\left|\sigma_{y}\right| \rightarrow\right\rangle=0 \quad\left\langle\leftarrow\left|\sigma_{y}\right| \rightarrow\right\rangle=1 .
\end{array}
$$

This implies that for magnetization parallel to the current direction, backscattering is due to majority-to-minority and minority-to-majority band transitions while for magnetization perpendicular to the current, allowed transitions are the majority-to-majority and minority-to-minority. The lowresistivity and high-resistivity magnetization orientations therefore switched places compared to the Rashba model and the AMR becomes negative. 
The spin texture of the Dresselhaus model is not circularly symmetric, however. It evolves from radial for $\mathbf{k}$ parallel to the [100] or [010] crystal axes to tangential for $\mathbf{k}$ parallel to the [110] or [110] diagonals, as shown in Fig. 2(a). The backscattering amplitudes for current along the diagonal, highlighted in Fig. 2(c), are hence identical as in the Rashba model, implying positive AMR for this current direction. The lower symmetry of the Dresselhaus model does not give rise to anisotropy in the resistivity of the system in the absence of magnetization. ${ }^{14}$ However, when magnetization is present the system acquires a crystalline AMR which reflects the underlying cubic symmetry of the spin texture. We remark that both the negative and positive AMRs of the Dresselhaus model vanish in the limit of $k_{+} \approx k_{-}$. Also in analogy with the behavior of the Rashba model, the AMRs with the respective signs are recovered in this limit when the pure magnetic impurity potential is replaced with the combined electromagnetic potential (see Sec. III and Fig. 6).

\section{AMR in the Rashba-Dresselhaus model with $|\boldsymbol{\alpha}|=|\boldsymbol{\beta}|$}

We now briefly comment on the potential importance of long-range nature of the impurity potential on the basic AMR phenomenology. For the demonstration of this effect, a singular model combining Rashba and Dresselhaus SOIs with $|\alpha|=|\beta|$ is particularly suitable. The Hamiltonian containing Rashba and Dresselhaus spin-orbit coupling terms of equal strength has singular properties ${ }^{33-35}$ (in particular additional symmetries). The internal spin-orbit coupling field has a $\mathbf{k}$-vector independent orientation (along the [1피-axis for $\alpha=\beta$ ). Spins on one circular Fermi contour are aligned parallel to this field while on the other contour they take the antiparallel alignment. Additionally, as shown in Fig. 3(a), this singular SOI shifts the two equal-size Fermi contours with respect to each other along a direction perpendicular to the direction of the spin-orbit field.

Because of the rigid spin-texture of the $|\alpha|=|\beta|$ RashbaDresselhaus model on two mutually shifted but otherwise identical circular Fermi contours, the backscattering amplitudes for a short-range impurity potential are independent of both the relative angle between $\mathbf{M}$ and the group velocity of the state moving along $\mathbf{I}$, and of the direction of current with respect to crystal axes. The AMR therefore completely vanishes in this model. Nevertheless, Figs. 3(b) and 3(c) illustrate that the AMR, including its crystalline component, is recovered when the scattering amplitudes pick up a dependence on the transferred momentum, i.e., for impurities carrying a long-range electromagnetic potential.

\section{AMR in the spherical Kohn-Luttinger model}

We conclude our excursion into the basic phenomenology of AMR, produced by scattering of spin-orbit coupled carriers from polarized magnetic impurities, by considering higher spin state of the carriers. We show that seemingly identical spin textures can result in opposite sign of the AMR for spin-1/2 and higher spin carriers, and argue that the AMR can have opposite sign when carriers with higher spin are

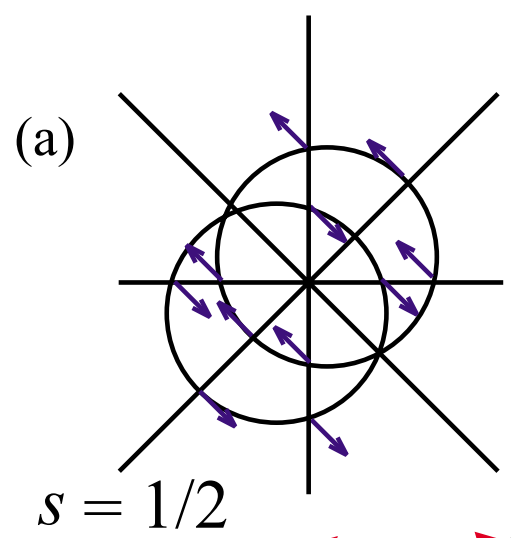

(b)

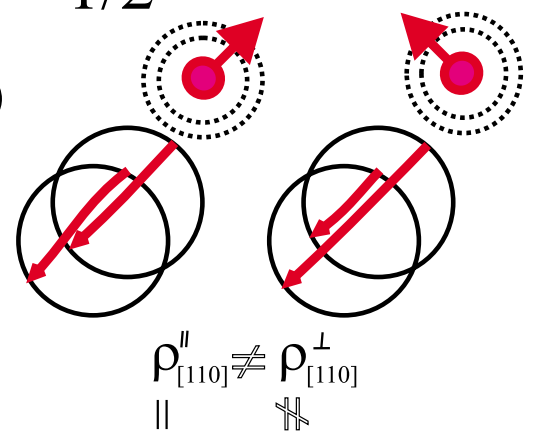

(c)

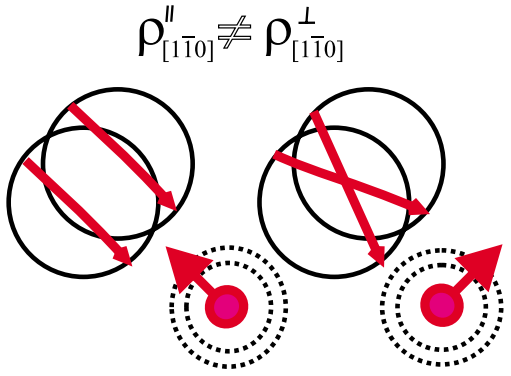

FIG. 3. (Color online) (a) Spin texture along the Fermi contours of Rashba-Dresselhaus model with $\alpha=\beta$. The AMR is zero for any type of short-range impurities. However, for long-range magnetic impurities the scattering amplitudes depend on the momentum transfer (illustrated by the length of the arrows) and nonzero AMR arises for current both along (b) [110] and (c) [ 110$]$ crystallographic directions.

scattered from a pure magnetic or from a combined electromagnetic potential. Again seeking the minimal SOI model on which this AMR phenomenology can be demonstrated without performing detailed transport calculations we choose the four-band spherical three-dimensional Kohn-Luttinger Hamiltonian for total angular momentum $j=3 / 2$ carriers,

$$
H_{K L}=\frac{\hbar^{2}}{2 m}\left[\left(\gamma_{1}+\frac{5}{2} \gamma_{2}\right) k^{2}-2 \gamma_{2}(\mathbf{k} \cdot \mathbf{j})^{2}\right]+h j_{z}
$$

with $h \rightarrow 0$. The $k_{x}, k_{y}$ plane (with infinitesimal $k_{z}$ ) spintextures depicted in Fig. 4(a) are obtained by realizing that the spin operator $\mathbf{s}=\mathbf{j} / 3$ in the four-band model, by defining the momentum quantization axes parallel to $\mathbf{k}$, and considering only the $j_{\mathbf{k}}= \pm 3 / 2$ bands (heavy holes). The infinitesimal exchange field $h$ in Eq. (6) is included to lift the degeneracy of these two bands, and $\gamma_{1}$ and $\gamma_{2}$ are the Luttinger 

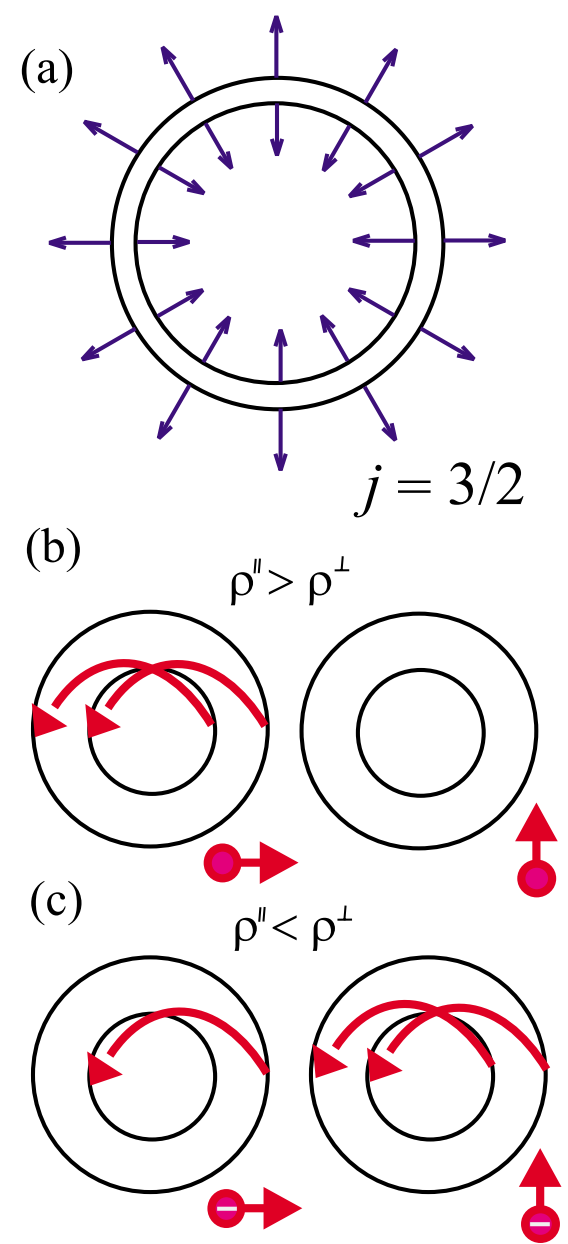

FIG. 4. (Color online) (a) Cross-section (parallel to the $k_{x}, k_{y}$ plane) of the 3D radial spin texture belonging to the two lowerenergy bands of the Kohn-Luttinger Hamiltonian. The two Fermi surfaces are sketched with different sizes for clarity, although the Hamiltonian (6) implies $k_{-}=k_{+}$as $h \rightarrow 0$. (b) Dominant scattering channels for magnetic impurities, note the difference to Fig. 2(b). (c) The same as (b) for electromagnetic scatterers.

parameters specific to the particular semiconductor valence bands for which $H_{K L}$ is derived from the conventional $\mathbf{k} \cdot \mathbf{p}$ approximation. ${ }^{22,36}$

Unlike the spin-1/2 Dresselhaus model, the radial spin texture in the $j=3 / 2$ Kohn-Luttinger model yields a positive AMR for purely magnetic scatterers. This can be illustrated using an analogous representation as in Eqs. (5) to relate the scattering amplitudes for impurity potential $\propto \hat{e}_{\mathbf{M}} \cdot \mathbf{S} / s$ $=\hat{e}_{\mathbf{M}} \cdot \mathbf{j} / j$ and the spin texture. For the $j=3 / 2$ carriers we obtain $^{37}$

$$
\begin{aligned}
& \left\langle\rightarrow\left|j_{x}\right| \rightarrow\right\rangle \neq 0 \quad\left\langle\leftarrow\left|j_{x}\right| \rightarrow\right\rangle=0, \\
& \left\langle\rightarrow\left|j_{y}\right| \rightarrow\right\rangle=0 \quad\left\langle\leftarrow\left|j_{y}\right| \rightarrow\right\rangle=0 .
\end{aligned}
$$

This implies, as highlighted in Fig. 4(b), that for magnetization parallel to the current direction, backscattering is due to majority-to-minority and minority-to-majority band transitions as in the case of spin-1/2 carriers. However, for magnetization perpendicular to the current, there are no allowed backscattering transitions in contrast to the spin-1/2 Dresselhaus model in Fig. 2(b). This makes now the latter configuration the low-resistivity state and AMR for the radial spin texture of the Kohn-Luttinger model becomes positive for pure magnetic impurity potential even for $k_{+} \approx k_{-}$. Boltzmann equation calculation of the AMR presented in Appendix B (and also an independent calculation based on the Green's function formalism ${ }^{23}$ ) again confirm our heuristic conclusion of Fig. 4(b).

On the other hand, electromagnetic scatterers $\propto \frac{3}{2} \rrbracket+j_{x, y}$ produce negative AMR in the Kohn-Luttinger model ${ }^{37,38}$ in the very same way as it is shown in Fig. 6(b) for the Dresselhaus model, and in both cases, this behavior can again be inferred using relations (7) and (5) with $j_{x, y}$ and $\sigma_{x, y}$ replaced by $\frac{3}{2} 1+j_{x, y}$ and $1+\sigma_{x, y}$, respectively. Dominant scattering channels which suggest that $\mathrm{AMR}<0$ are summarized in Fig. 4(c). Contrary to the Dresselhaus model (4), the SOI of the Kohn-Luttinger model (6) in combination with polarized scatterers therefore can produce AMR of either sign, e.g., depending on the carrier-density-controlled screening of the impurities. ${ }^{37,38}$ This qualitative difference between Dresselhaus and Kohn-Luttinger models highlights the fact that knowledge of spin textures, such as Figs. 2(a) or 4(a), may not be sufficient to analyze the scattering properties of the model and appropriate matrix elements such as Eqs. (5) or (7) should always be verified.

\section{QUANTITATIVE RESULTS FOR THE AMR IN THE RASHBA-DRESSELHAUS MODEL}

The AMR analysis in the previous section utilizes the RTA (in fact only the backscattering term of the RTA) which, in general, is not a rigorous theory approach for anisotropic systems. ${ }^{32}$ It is therefore desirable to calculate the AMR beyond the RTA, not only to obtain quantitative predictions but also to confirm the validity of the basic AMR phenomenology inferred above. As in Sec. II, we will employ the firstorder Born approximation for calculating the scattering probabilities but will solve the corresponding integral Boltzmann equation exactly. To provide better physical insight we start with explaining the relation between the RTA and the full semiclassical Boltzmann theory for the two-dimensional SOI systems. Exact analytical solutions to the Boltzmann equation are then derived for Rashba and Dresselhaus model with short range electromagnetic impurity potentials and for the combined Rashba-Dresselhaus model with arbitrary $\alpha$ and $\beta$ and with magnetic impurities.

\section{A. Relation between RTA and integral Boltzmann equation in the Rashba model}

Because the equilibrium Fermi distribution $f^{0}\left(E_{i, \mathbf{k}}\right)$ is a function only of energy, we can write the Boltzmann equation $^{32}$ in $d=2$ dimensions as 


$$
\begin{aligned}
-|e| \mathbf{E} \cdot \mathbf{v}_{i, \mathbf{k}} \frac{\partial f^{0}\left(E_{i, \mathbf{k}}\right)}{\partial E_{i, \mathbf{k}}}= & -\int \frac{d^{d} k^{\prime}}{(2 \pi)^{d}} \sum_{i^{\prime}} w\left(i, \mathbf{k} ; i^{\prime}, \mathbf{k}^{\prime}\right) \delta\left(E_{i^{\prime}, \mathbf{k}^{\prime}}-E_{i, \mathbf{k}}\right)\left[f(i, \mathbf{k})-f\left(i^{\prime}, \mathbf{k}^{\prime}\right)\right] \\
= & -\left[f(i, \mathbf{k})-f^{0}\left(E_{i, \mathbf{k}}\right)\right] \sum_{i^{\prime}} \int \frac{d^{d} k^{\prime}}{(2 \pi)^{d}} w\left(i, \mathbf{k} ; i^{\prime}, \mathbf{k}^{\prime}\right) \delta\left(E_{i^{\prime}, \mathbf{k}^{\prime}}-E_{i, \mathbf{k}}\right) \\
& +\int \frac{d^{d} k^{\prime}}{(2 \pi)^{d}} \sum_{i^{\prime}} w\left(i, \mathbf{k} ; i^{\prime}, \mathbf{k}^{\prime}\right) \delta\left(E_{i^{\prime}, \mathbf{k}^{\prime}}-E_{i, \mathbf{k}}\right)\left[f\left(i^{\prime}, \mathbf{k}^{\prime}\right)-f^{0}\left(E_{i^{\prime}, \mathbf{k}^{\prime}}\right)\right],
\end{aligned}
$$

where $\mathbf{v}_{i, \mathbf{k}}=\partial E_{i, \mathbf{k}} / \partial \hbar \mathbf{k}$ is the group velocity, $f(i, \mathbf{k})$ is the nonequilibrium distribution function, and $i= \pm$ is the band index. The transition probabilities in the first-order Born approximation are given by

$$
w\left(i, \mathbf{k} ; i^{\prime}, \mathbf{k}^{\prime}\right)=\frac{2 \pi n}{\hbar}\left|\left\langle i, \mathbf{k}|V| i^{\prime}, \mathbf{k}^{\prime}\right\rangle\right|^{2},
$$

where $V$ is the strength of the short-range scattering potential of impurities with density $n$. Energy conservation during elastic scattering processes was already incorporated into the right-hand side of Eq. (8).

In the Rashba model, $\Sigma_{i^{\prime}} w\left(i, \mathbf{k} ; i^{\prime}, \mathbf{k}^{\prime}\right)$ is a constant ${ }^{51}$ for a short-range electric potential, $V \propto 1$, or magnetic potential, $V \propto \hat{e}_{M} \cdot \boldsymbol{\sigma}$. In the limit of nearly degenerate bands, $E_{i, \mathbf{k}}$ $\approx E_{i^{\prime}, \mathbf{k}}$, we can find a solution of Eq. (8) in the RTA form,

$$
f(i, \mathbf{k})-f^{0}\left(E_{i, \mathbf{k}}\right)=c|e| \mathbf{E} \cdot \mathbf{v}_{i, \mathbf{k}} \frac{\partial f^{0}\left(E_{i, \mathbf{k}}\right)}{\partial E_{i, \mathbf{k}}} .
$$

Plugged in Eq. (8), the second term on the right-hand side drops out because of the independence of $\Sigma_{i^{\prime}} w\left(i, \mathbf{k} ; i^{\prime}, \mathbf{k}^{\prime}\right)$ on $\mathbf{k}^{\prime}$ and because the group velocity averages to zero over the Fermi contour, and the first term gives

$$
\frac{1}{c}=\int \frac{d^{2} k^{\prime}}{(2 \pi)^{2}} \sum_{i^{\prime}} w\left(i, \mathbf{k} ; i^{\prime}, \mathbf{k}^{\prime}\right) \delta\left(E_{i^{\prime}, \mathbf{k}^{\prime}}-E_{i, \mathbf{k}}\right) \equiv \frac{1}{\tau} .
$$

The electrical current within the semiclassical linear response, given by

$$
\mathbf{j}=-e \sum_{i} \int \frac{d^{2} k}{(2 \pi)^{2}} \mathbf{v}_{i, \mathbf{k}}\left[f(i, \mathbf{k})-f^{0}(i, \mathbf{k})\right],
$$

is exactly proportional to the quasiparticle broadening lifetime $\tau$ in this case. Same RTA form of the Boltzmann equation applies also to the Rashba-Dresselhaus model with $|\alpha|$ $=|\beta|$ because the rigid spin-texture of this singular case implies constant transition probabilities for any short-range electromagnetic potential.

In the Rashba model with nondegenerate bands, $E_{i, \mathbf{k}}$ $\neq E_{i^{\prime}, \mathbf{k}}$, the RTA solution (10) to the Boltzmann equation can still be found for a nonmagnetic potential, $V \propto 1$. The scattering probability $w\left(i, \mathbf{k} ; i^{\prime}, \mathbf{k}^{\prime}\right)$ depends in this case on the magnitude of the transition angle, $\left|\theta-\theta^{\prime}\right|$. It implies that from the product,

$$
\begin{aligned}
\mathbf{E} \cdot \mathbf{v}_{i^{\prime}, \mathbf{k}^{\prime}}= & v_{i} \mathbf{E} \cdot \mathbf{v}_{\mathbf{k}} \frac{v_{i^{\prime}}}{v_{i}} \cos \left(\theta-\theta^{\prime}\right) \\
& +v_{i}(\hat{\mathbf{z}} \times \mathbf{E}) \cdot \mathbf{v}_{\mathbf{k}} \frac{v_{i^{\prime}}}{v_{i}} \sin \left(\theta-\theta^{\prime}\right),
\end{aligned}
$$

the transverse term $\propto \sin \left(\theta-\theta^{\prime}\right)$ does not contribute to the second term on the right-hand side of Eq. (8). The longitudinal term $\propto \cos \left(\theta-\theta^{\prime}\right)$ contributes to Eq. (8) and the Boltzmann equation takes a modified RTA form with

$$
\begin{aligned}
\frac{1}{c}= & \int \frac{d^{2} k^{\prime}}{(2 \pi)^{2}} \sum_{i^{\prime}} w\left(i, \mathbf{k} ; i^{\prime}, \mathbf{k}^{\prime}\right) \delta\left(E_{i^{\prime}, \mathbf{k}^{\prime}}-E_{i, \mathbf{k}}\right) \\
& \times\left[1-\frac{v_{i^{\prime}}}{v_{i}} \cos \left(\theta-\theta^{\prime}\right)\right] \equiv \frac{1}{\tau_{t r}} .
\end{aligned}
$$

Electrical current is now proportional to the transport lifetime which gives larger weight to larger angle scattering transitions.

The transport lifetime form of the Boltzmann equation has been the basis of qualitative discussions in Sec. II where we further simplified the analysis by considering only the leading contribution to current in Eq. (12) from states with $\mathbf{v}_{i, \mathbf{k}} \| \mathbf{E}$. For all spin-textures and orientations of $\mathbf{E}$ and $\mathbf{M}$ considered in Sec. II, $w\left(i, \mathbf{k} ; i^{\prime}, \mathbf{k}^{\prime}\right)$ depends only on $\left|\theta-\theta^{\prime}\right|$ for the special $\mathbf{k}$ states with group velocity parallel to the electric field. This justifies the internal consistency of the RTA based analyses in Sec. II and explains their qualitative validity.

\section{B. Solution to the Boltzmann equation for the Rashba- Dresselhaus model}

To obtain quantitative AMR predictions we need to perform the full $\mathbf{k}$-space integration in the expression (12) for the electrical current. For arbitrary k-vector and other than the few special cases discussed in the previous subsection (which all happen to give zero AMR), the integral of the transverse term in Eq. (13) may not vanish and/or the integrated scattering probability in the first term on the righthand side of Eq. (8) may not be independent of k. In these cases the RTA form of the solution to the Boltzmann equation fails. For the Rashba-Dresselhaus model we can, nevertheless, find the exact solution to the Boltzmann equation in an analytic form which allows us to directly compare the 
TABLE I. Conductivity tensor for a 2DEG confined in a [001]-grown III-V semiconductor heterostructure at different magnetization of scatterers. Here, $\sigma_{0}=e^{2} n_{e} \tau / m$, see also Eq. (A7), $n_{e}$ is the electron density, see Eq. (A8), and $A / \alpha^{2}=B / \beta^{2}=e^{2} m \tau /\left(\pi \hbar^{4}\right)$. The conductivity corrections depend essentially on the type of spin-orbit interactions which is either Rashba $(\alpha)$ or Dresselhaus $(\beta)$ one. The conductivity expressions for arbitrary $\alpha$ and $\beta$ can be found in Appendix A.

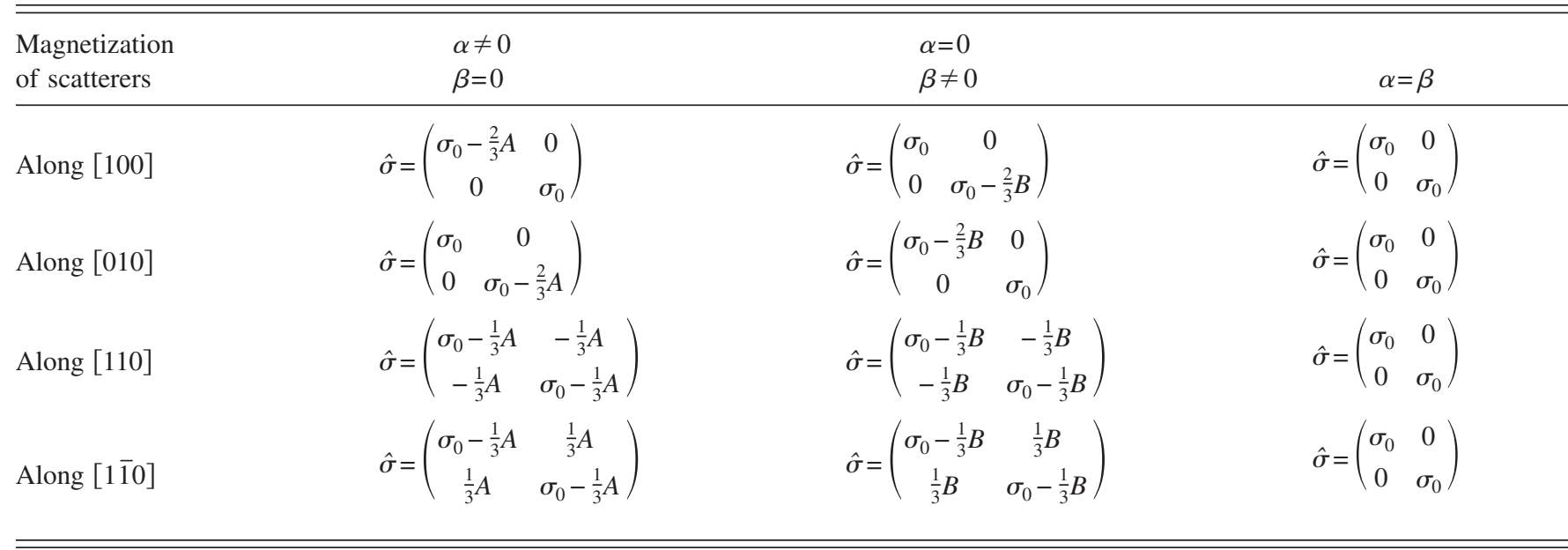

corresponding quantitative AMR predictions with the qualitative results of Sec. II.

The method has been previously derived ${ }^{32}$ for pure Rashba model in which the angular dependence of the scattering probability function for the short-range magnetic potential, e.g., $V \propto \sigma_{x}$, is given by

$$
w\left(i, \theta ; i^{\prime}, \theta^{\prime}\right) \propto 1-i i^{\prime}\left(\cos \theta \cos \theta^{\prime}-\sin \theta \sin \theta^{\prime}\right) .
$$

Since also $\int_{0}^{2 \pi} d \theta^{\prime} w\left(i, \theta ; i^{\prime}, \theta^{\prime}\right)$ is a constant independent of $\theta$, the first term on the right-hand side of Eq. (8) implies that $f(i, \mathbf{k})-f^{0}\left(E_{i, \mathbf{k}}\right)$ must contain term $\mathbf{E} \cdot \mathbf{v}_{i, k}(\theta)$ and the second term on the right-hand side of Eq. (8) implies that $f(i, \mathbf{k})$ $-f^{0}\left(E_{i, \mathbf{k}}\right)$ must contain harmonics of $w\left(i, \theta ; i^{\prime}, \theta^{\prime}\right)$ which in both cases happen to be just $\cos \theta$ and $\sin \theta$. No higher order Fourier components can contribute to the nonequilibrium distribution function in this case and Eq. (8) can be solved analytically.

The AMR of the Rashba model with magnetic impurity potential is summarized in the first column of Table I and also plotted in Fig. 5 as a function of the ratio $E_{F} \hbar^{2} /\left(m \alpha^{2}\right)$. Here $E_{F}=0$ corresponds to the minority Rashba band being just depleted and $E_{F} \hbar^{2} /\left(m \alpha^{2}\right) \gg 1$ to nearly degenerate $i$ $= \pm$ Rashba bands. Consistent with the qualitative results of Sec. II we find a positive AMR which vanishes as the radii of the minority and majority band Fermi contours approach each other.

For Rashba model with the electromagnetic potential, e.g., $V \propto 1+\sigma_{x}$, the integral $\int_{0}^{2 \pi} d \theta^{\prime} w\left(i, \theta ; i^{\prime}, \theta^{\prime}\right) \propto 1+i i^{\prime} \sin \theta$ is not a constant which implies the presence of higher order Fourier components in $f(i, \mathbf{k})-f^{0}\left(E_{i, \mathbf{k}}\right)$. Still an analytical form can be found for the distribution function, see the note added in proof of Ref. 32. Analogous arguments apply also to the Dresselhaus model with electromagnetic impurities. The dependence of AMRs in the two models as a function of the ratio $a$ of the electrical and magnetic parts of the impurity potential $V \propto a \rrbracket+\hat{e}_{M} \cdot \boldsymbol{\sigma}$ in the limit of nearly degenerate bands and for current along the [100] axis is given by

$$
\mathrm{AMR}= \begin{cases} \pm 2 a^{2}, & \text { for }|a| \leq 1 \\ \pm 2 / a^{2}, & \text { for }|a| \geq 1,\end{cases}
$$

where +/ - corresponds to the Rashba/Dresselhaus model. For illustration, we also plot the result in Fig. 6. Again in full qualitative agreement with the analysis in Sec II, the AMRs in both models are zero for $a=0$. They also vanish in the limit of $a \rightarrow \infty$ since no AMR occurs if the system is not magnetically polarized. For intermediate ratios of the strengths of the electric and magnetic parts of the potential, a positive AMR in the Rashba model reflects the tangential spin-1/2 texture while the negative AMR in the Dresselhaus model reflects the radial texture of the states with large group velocity projection to the direction of the current. The singular peak at $a=1$ originates from the coherent superposition of nonmagnetic and magnetic scattering amplitudes which results in zero scattering probability of one of the two states moving along the current direction, ${ }^{52}$ as we already pointed out in Sec II and illustrated in Fig. 1(c).
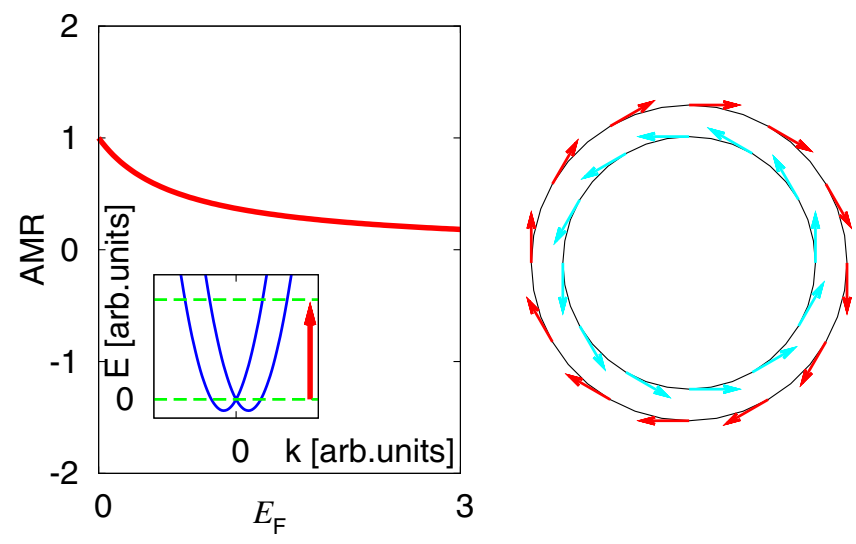

FIG. 5. (Color online) Pure Rashba system with magnetic impurity, AMR as a function of the Fermi energy $E_{F}$ in units of $m \alpha^{2} / \hbar^{2}$ 

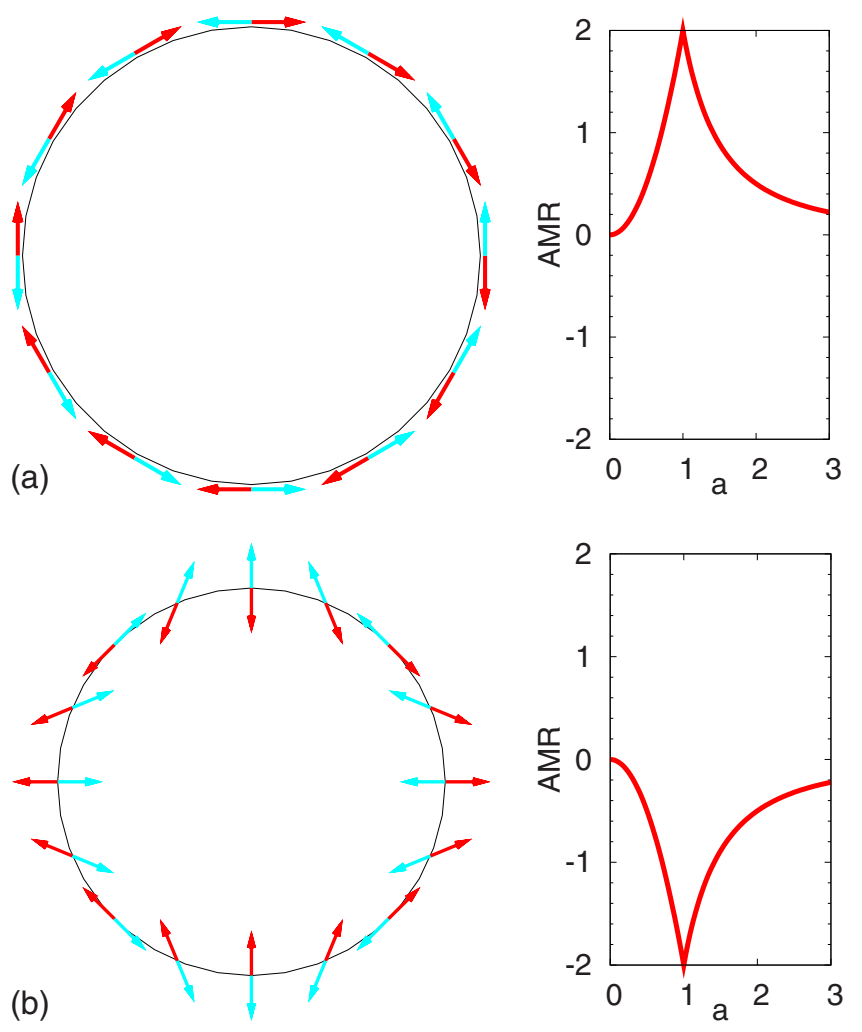

FIG. 6. (Color online) AMR for current flowing along [100] crystal axis in a pure (a) Rashba and (b) Dresselhaus systems with electromagnetic impurity $\left(\propto a \rrbracket+\sigma_{x, y}\right)$, varying $a$, the ratio between the electric and magnetic part of the potential. The Fermi energy $E_{F}$ is taken much larger than the spin-orbit interaction, so that the Fermi radii of the two bands become almost equal, see details in text.

In Table I, we included conductivity components obtained from the exact solution to the Boltzmann equation for Rashba and Dresselhaus models and the magnetic potential with $\mathbf{M}$ oriented along the main in-plane crystal axes and along the in-plane diagonals (derived as shown below). The component $\sigma_{11}$ in the table corresponds to the longitudinal response to $\mathbf{E}$ along the [100] axis and $\sigma_{22}$ along the [010] axis. To obtain AMR values for electric field along an arbitrary angle $\phi$ measured from the [100] axis the conductivity tensors with appropriate magnetization direction of scatterers have to be rotated by $R_{-\phi} \hat{\sigma} R_{\phi}$ where the rotation matrix is given by

$$
R_{\phi}=\left(\begin{array}{cc}
\cos \phi & -\sin \phi \\
\sin \phi & \cos \phi
\end{array}\right) .
$$

The AMR as defined in Eq. (1) is independent of $\phi$ in the Rashba model confirming the absence of crystalline AMR components in this system. In the Dresselhaus model, AMRs of opposite sign are obtained for current along the main inplane axes $(\phi=0, \pi / 2)$ and along the diagonals $(\phi$ $=\pi / 4,3 \pi / 4)$, consistent with the crystalline nature of the AMR inferred in Sec II. A closer inspection of the full angular dependence of the AMR in the Rashba and Dresselhaus models allows us to relate our quantitative microscopic re-
TABLE II. Magnetization-direction-dependent factors $P^{\hat{e}_{M}}$ of Eq. (21) relevant for magnetic impurities. Functions $\cos \gamma_{k}$, $\sin \gamma_{k}$ are given in the main text.

\begin{tabular}{lc}
\hline \hline$\hat{e}_{M}$ & $P^{\hat{e}_{M}\left(i, \theta ; i^{\prime}, \theta^{\prime}\right)}$ \\
\hline$[100]$ & $1+i i^{\prime} \cos \left(\gamma_{k}+\gamma_{k^{\prime}}\right)$ \\
{$[010]$} & $1-i i^{\prime} \cos \left(\gamma_{k}+\gamma_{k^{\prime}}\right)$ \\
{$[110]$} & $1-i i^{\prime} \sin \left(\gamma_{k}+\gamma_{k^{\prime}}\right)$ \\
{$[1 \overline{1} 0]$} & $1+i i^{\prime} \sin \left(\gamma_{k}+\gamma_{k^{\prime}}\right)$ \\
{$[001]$} & $1-i i^{\prime} \cos \left(\gamma_{k}-\gamma_{k^{\prime}}\right)$ \\
\hline \hline
\end{tabular}

sults to the standard phenomenology of the angle-dependent longitudinal resistivity for systems with cubic anisotropies, ${ }^{29}$

$$
\begin{aligned}
\rho(\omega, \phi) / \rho_{a v}-1= & C_{I} \cos 2(\omega-\phi)+C_{I, c} \cos 2(\omega+\phi) \\
& +C_{c} \cos 4 \omega,
\end{aligned}
$$

where $\omega$ and $\phi$ denote the direction angles of $\mathbf{M}$ and $\mathbf{E}$ to the [100] crystal axis, respectively, and $\rho_{a v}$ is the average resistivity over all magnetization directions. The coefficient $C_{I}$ of the noncrystalline AMR component, which depends only on the relative angle between current and magnetization, equals $1 / 3$ for the Rashba model and 0 for the Dresselhaus model. The coefficient $C_{I, c}$ of the first crystalline component is nonzero (equals $-1 / 3$ ) in the Dresselhaus model and zero in the Rashba model, consistent with the crystalline nature of the AMR in the Dresselhaus SOI system and noncrystalline AMR of the Rashba system. The coefficient $C_{c}$ of the higher order crystalline term is zero in both models.

We conclude this section by presenting the exact solution to the Boltzmann equation and the corresponding AMR values for the combined Rashba-Dresselhaus model which implies the dispersion law $E_{ \pm, \mathbf{k}}=\frac{\hbar^{2} k^{2}}{2 m} \pm k \kappa_{\theta}$, where $\kappa_{\theta}$ $=\sqrt{\alpha^{2}+\beta^{2}+2 \alpha \beta \sin 2 \theta}$ is the $\theta$-dependent subband spin splitting. We consider a general case of arbitrary $\alpha$ and $\beta$ but restrict ourselves to the pure magnetic impurity potential. The electron group velocity $(1 / \hbar) \nabla_{\mathbf{k}} E_{\mathbf{k} \pm}$ is now anisotropic and given by

$$
\begin{aligned}
& \left.\mathbf{v}_{ \pm, k}\right|_{x}=\hbar k_{x} / m \pm\left(\beta \cos \gamma_{k}+\alpha \sin \gamma_{k}\right) / \hbar, \\
& \left.\mathbf{v}_{ \pm, k}\right|_{y}=\hbar k_{y} / m \pm\left(\alpha \cos \gamma_{k}+\beta \sin \gamma_{k}\right) / \hbar,
\end{aligned}
$$

with $k_{x}=k \cos \theta, \quad k_{y}=k \sin \theta, \quad$ and $\quad \sin \gamma_{k}=(\alpha \cos \theta$ $+\beta \sin \theta) / \kappa_{\theta}, \cos \gamma_{k}=(\beta \cos \theta+\alpha \sin \theta) / \kappa_{\theta}$.

The derivation relies on vanishing angular integrals of the generating functions of $w\left(i, \mathbf{k} ; i^{\prime}, \mathbf{k}^{\prime}\right) \propto P\left(i, \theta ; i^{\prime}, \theta^{\prime}\right)$ (summarized in Table II for $\mathbf{M}$ along the main in-plane crystal axes and the in-plane diagonals) which are $\cos \theta / \kappa_{\theta}$ and $\sin \theta / \kappa_{\theta}$. As in the case of the Rashba model discussed above, the independence of $\int_{0}^{2 \pi} d \theta^{\prime} w\left(i, \theta ; i^{\prime}, \theta^{\prime}\right)$ on $\theta$ implies that the nonequilibrium distribution function contains only the group velocity, see Eqs. (19) and (20), and the generating functions of $P\left(i, \theta ; i^{\prime}, \theta^{\prime}\right)$ which are $\cos \theta / \kappa_{\theta}$ and $\sin \theta / \kappa_{\theta}$. Note that for arbitrary $\alpha$ and $\beta$ and for the orientations of $\mathbf{M}$ considered in Tables I and II the transition probabilities can then be written as 
TABLE III. Anisotropic part $\widehat{\sigma_{1}}$ of the total conductivity tensor $\hat{\sigma}=1 \sigma_{0}+\widehat{\sigma_{1}}$ for arbitrary $\alpha$ and $\beta$.

Magnetization

direction

$\alpha \geq \beta$

[100]

[010]

$$
\begin{aligned}
& \left(\begin{array}{cc}
-\frac{2 e^{2} m \alpha^{2}\left(\alpha^{2}-\beta^{2}\right) \tau}{\hbar^{4} \pi\left(3 \alpha^{2}+\beta^{2}\right)} & -\frac{2 e^{2} m \alpha\left(\alpha^{2}-\beta^{2}\right) \beta \tau}{\hbar^{4} \pi\left(3 \alpha^{2}+\beta^{2}\right)} \\
-\frac{2 e^{2} m \alpha\left(\alpha^{2}-\beta^{2}\right) \beta \tau}{\hbar^{4} \pi\left(3 \alpha^{2}+\beta^{2}\right)} & -\frac{2 e^{2} m \beta^{2}\left(\alpha^{2}-\beta^{2}\right) \tau}{\hbar^{4} \pi\left(3 \alpha^{2}+\beta^{2}\right)}
\end{array}\right) \\
& \left(\begin{array}{ll}
-\frac{2 e^{2} m \beta^{2}\left(\alpha^{2}-\beta^{2}\right) \tau}{\hbar^{4} \pi\left(3 \alpha^{2}+\beta^{2}\right)} & -\frac{2 e^{2} m \alpha\left(\alpha^{2}-\beta^{2}\right) \beta \tau}{\hbar^{4} \pi\left(3 \alpha^{2}+\beta^{2}\right)} \\
-\frac{2 e^{2} m \alpha\left(\alpha^{2}-\beta^{2}\right) \beta \tau}{\hbar^{4} \pi\left(3 \alpha^{2}+\beta^{2}\right)} & -\frac{2 e^{2} m \alpha^{2}\left(\alpha^{2}-\beta^{2}\right) \tau}{\hbar^{4} \pi\left(3 \alpha^{2}+\beta^{2}\right)}
\end{array}\right) \\
& \left(\begin{array}{ll}
-\frac{e^{2} m(\alpha-\beta)(\alpha+\beta)^{2} \tau}{\hbar^{4} \pi(3 \alpha+\beta)} & -\frac{e^{2} m(\alpha-\beta)(\alpha+\beta)^{2} \tau}{\hbar^{4} \pi(3 \alpha+\beta)} \\
-\frac{e^{2} m(\alpha-\beta)(\alpha+\beta)^{2} \tau}{\hbar^{4} \pi(3 \alpha+\beta)} & -\frac{e^{2} m(\alpha-\beta)(\alpha+\beta)^{2} \tau}{\hbar^{4} \pi(3 \alpha+\beta)}
\end{array}\right) \\
& \left(\begin{array}{cc}
-\frac{e^{2} m(\alpha-\beta)(\alpha+\beta)^{2} \tau}{\hbar^{4} \pi(3 \alpha+\beta)} & \frac{e^{2} m(\alpha-\beta)(\alpha+\beta)^{2} \tau}{\hbar^{4} \pi(3 \alpha+\beta)} \\
\frac{e^{2} m(\alpha-\beta)(\alpha+\beta)^{2} \tau}{\hbar^{4} \pi(3 \alpha+\beta)} & -\frac{e^{2} m(\alpha-\beta)(\alpha+\beta)^{2} \tau}{\hbar^{4} \pi(3 \alpha+\beta)}
\end{array}\right)
\end{aligned}
$$

$$
\begin{gathered}
\beta \geq \alpha \\
\left(\begin{array}{cc}
\frac{2 e^{2} m \alpha^{2}\left(\alpha^{2}-\beta^{2}\right) \tau}{\hbar^{4} \pi\left(\alpha^{2}+3 \beta^{2}\right)} & \frac{2 e^{2} m \alpha\left(\alpha^{2}-\beta^{2}\right) \beta \tau}{\hbar^{4} \pi\left(\alpha^{2}+3 \beta^{2}\right)} \\
\frac{2 e^{2} m \alpha\left(\alpha^{2}-\beta^{2}\right) \beta \tau}{\hbar^{4} \pi\left(\alpha^{2}+3 \beta^{2}\right)} & \frac{2 e^{2} m\left(\alpha^{2}-\beta^{2}\right) \beta^{2} \tau}{\hbar^{4} \pi\left(\alpha^{2}+3 \beta^{2}\right)}
\end{array}\right) \\
\left(\begin{array}{ll}
\frac{2 e^{2} m\left(\alpha^{2}-\beta^{2}\right) \beta^{2} \tau}{\hbar^{4} \pi\left(\alpha^{2}+3 \beta^{2}\right)} & \frac{2 e^{2} m \alpha\left(\alpha^{2}-\beta^{2}\right) \beta \tau}{\hbar^{4} \pi\left(\alpha^{2}+3 \beta^{2}\right)} \\
\frac{2 e^{2} m \alpha\left(\alpha^{2}-\beta^{2}\right) \beta \tau}{\hbar^{4} \pi\left(\alpha^{2}+3 \beta^{2}\right)} & \frac{2 e^{2} m \alpha^{2}\left(\alpha^{2}-\beta^{2}\right) \tau}{\hbar^{4} \pi\left(\alpha^{2}+3 \beta^{2}\right)}
\end{array}\right) \\
\left(\begin{array}{ll}
\frac{e^{2} m(\alpha-\beta)(\alpha+\beta)^{2} \tau}{\hbar^{4} \pi(\alpha+3 \beta)} & \frac{e^{2} m(\alpha-\beta)(\alpha+\beta)^{2} \tau}{\hbar^{4} \pi(\alpha+3 \beta)} \\
\frac{e^{2} m(\alpha-\beta)(\alpha+\beta)^{2} \tau}{\hbar^{4} \pi(\alpha+3 \beta)} & \frac{e^{2} m(\alpha-\beta)(\alpha+\beta)^{2} \tau}{\hbar^{4} \pi(\alpha+3 \beta)}
\end{array}\right) \\
\left(\begin{array}{cc}
\frac{e^{2} m(\alpha-\beta)(\alpha+\beta)^{2} \tau}{\hbar^{4} \pi(\alpha+3 \beta)} & -\frac{e^{2} m(\alpha-\beta)(\alpha+\beta)^{2} \tau}{\hbar^{4} \pi(\alpha+3 \beta)} \\
-\frac{e^{2} m(\alpha-\beta)(\alpha+\beta)^{2} \tau}{\hbar^{4} \pi(\alpha+3 \beta)} & \frac{e^{2} m(\alpha-\beta)(\alpha+\beta)^{2} \tau}{\hbar^{4} \pi(\alpha+3 \beta)}
\end{array}\right)
\end{gathered}
$$

$$
w\left(i, \mathbf{k} ; i^{\prime} \mathbf{k}^{\prime}\right)=\frac{1}{\nu \tau} P^{\hat{e}_{M}}\left(i, \theta ; i^{\prime} \theta^{\prime}\right),
$$

where $\nu=m / \pi \hbar^{2}$ is the density of states, the $\mathbf{k}$-vector independent constant $\tau$ is given by Eq. (11), and the angular probabilities $P^{\hat{e}} M\left(i, \theta ; i^{\prime} \theta^{\prime}\right)$ are explicitly written in Tab. II. The integral Boltzmann equation (8) is then solved by the distribution function of a form

$$
\begin{aligned}
f(i, \mathbf{k})-f^{0}\left(E_{i, \mathbf{k}}\right)= & \tau|e| \mathbf{E} \cdot \mathbf{v}_{i, \mathbf{k}} \frac{\partial f^{0}\left(E_{i, \mathbf{k}}\right)}{\partial E_{i, \mathbf{k}}} \\
& +\frac{\tau|e|}{\hbar} \frac{\partial f^{0}\left(E_{i, \mathbf{k}}\right)}{\partial E_{i, \mathbf{k}}}\left[\left(a_{x}^{\hat{e}_{M}} \frac{\cos \theta}{\kappa_{\theta}}+b_{x}^{\hat{e}_{M}} \frac{\sin \theta}{\kappa_{\theta}}\right) E_{x}\right. \\
& \left.+\left(a_{y}^{\hat{e}_{M}} \frac{\cos \theta}{\kappa_{\theta}}+b_{y}^{\hat{e}_{M}} \frac{\sin \theta}{\kappa_{\theta}}\right) E_{y}\right] .
\end{aligned}
$$

Values of the coefficients $a_{x, y}^{\hat{e}_{M}}, b_{x, y}^{\hat{e}_{M}}$ depend on the magnetization vector direction $\hat{e}_{M}$ and are given in Appendix A.

For $|\alpha|=|\beta|$, and general $\alpha, \beta$, analytical expressions for the conductivity tensor of the Rashba-Dresselhaus model and short-range magnetic impurity potential with $\mathbf{M}$ oriented along the main and diagonal in-plane axes can be found in Table I, and Table III in Appendix, respectively. As pointed out in Sec. II, the AMR vanishes for $|\alpha|=|\beta|$. For $\alpha \neq \beta$, however, the AMR is nonzero and depends both on the relative angle between current and magnetization and on the direction of current with respect to the crystallographic axes. The AMRs for various current directions can again be calculated by rotating the conductivity tensor given in Table III. For current along the [100]-axis, e.g., and $|\alpha| \geq|\beta|$ we obtain

$$
\mathrm{AMR}=\frac{2\left(1-r^{2}\right)^{2}}{2\left(1+r^{2}\right)^{2}+\left(3+r^{2}\right) \hbar^{2} E_{F} /\left(m \alpha^{2}\right)},
$$

where $r=\beta / \alpha$. In the opposite case of $|\alpha| \leq|\beta|$, the result is the same up to an exchange of $\alpha$ and $\beta$ in Eq. (23) and in the definition of $r$.
The smooth transition of the AMR from the pure Rashba to pure Dresselhaus model described by Eq. (23) is shown in Fig. 7 for $E_{F}=0$ and for intermediate $E_{F}$ corresponding to both majority and minority Rashba-Dresselhaus bands occupied. We point out that for $\alpha \neq \beta$ the AMR originates from not only the anisotropic spin texture on the Fermi contours but also, unlike the pure Rashba or pure Dresselhaus models, from anisotropic group velocities. In the special case of $|\alpha|$ $=|\beta|$, these two sources of anisotropy disappear and AMR vanishes for any short-range electromagnetic potential.

The relative displacement along the diagonal direction of the two circular Fermi contours is nevertheless a significant remaining imprint of the SOI in the band structure of the $|\alpha|=|\beta|$ model. The AMR can reappear if $w\left(i, \mathbf{k} ; i^{\prime}, \mathbf{k}^{\prime}\right)$ picks up a dependence on $\mathbf{k}$ and $\mathbf{k}^{\prime}$ due to other than the spintexture effect. As pointed out in Sec. II, a long-range (electro-)magnetic impurity potential combined with the two displaced Fermi circles would yield wave vector dependent $w\left(i, \mathbf{k} ; i^{\prime}, \mathbf{k}^{\prime}\right)$ and a nonzero AMR even for $|\alpha|=|\beta|$.

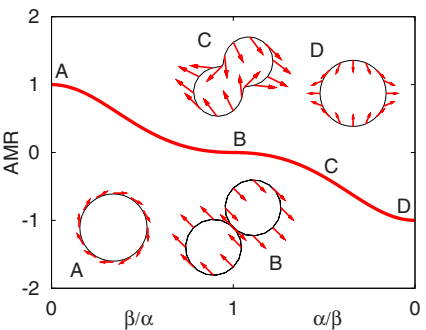

(a)

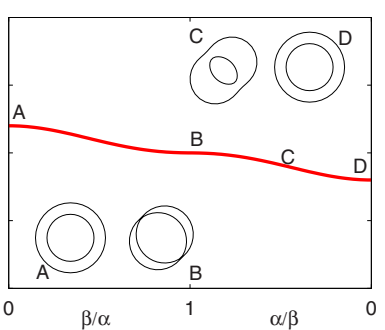

(b)
FIG. 7. (Color online) AMR for pure magnetic potential impurity as a function of the ratio $\alpha / \beta$. Pure Rashba (Dresselhaus) interaction corresponds to the left (right) edge. (a) Single band case, $E_{F}=0$ (very low electron concentration). Insets show the spin textures for several chosen values of $\alpha / \beta$. (b) Two band case with $E_{F} / \alpha^{2}>0$ fixed. Fermi lines are shown schematically, spin textures of the majority band are qualitatively similar to the single band case. In the limit $E_{F} \rightarrow \infty$, the AMR vanishes for any value of $\alpha / \beta$. 


\section{DISCUSSION}

Calculations in the previous sections show the following trends in the AMR: (i) for the Rashba-Dresselhaus model with a short-range magnetic impurity potential, the AMR is large $(100 \%)$ when the minority band is depleted and when the SOI is of a pure Rashba type $(\beta=0)$ or pure Dresselhaus type $(\alpha=0)$. (ii) The AMR vanishes when $|\alpha|=|\beta|$ or for an arbitrary $\alpha$ and $\beta$ when the majority and minority bands become nearly degenerate. (iii) For impurities containing a combined electromagnetic potential, the AMR has the same sign as for the pure magnetic impurity potential, is maximized when the two components have equal strength, and remains large $(200 \%)$ even in the limit of nearly degenerate Rashba-Dresselhaus bands. (iv) We have also noted (in agreement with Ref. 29) that in the higher-spin KohnLuttinger model, the AMR is expected to have opposite signs for pure magnetic potential and for electromagnetic potential with comparable strength of the two components. We will now discuss implications of observations (i-iii) and inspect the applicability of our linear-response quasiclassical theory for two-dimensional (2D) systems with realistic material parameters.

Two-dimensional electron systems with Rashba and Dresselhaus SOI have been studied in $n$-type InAs and GaAs quantum wells ${ }^{39-42}$ with mobilities $\mu$ up to $3 \times 10^{5}$ and 3.5 $\times 10^{6} \mathrm{~cm}^{2} / \mathrm{V} \mathrm{s}$, and magnitudes of the SOI of the order of $\sim 10^{-11}$ and $\sim 10^{-12} \mathrm{eV} \mathrm{m}$, respectively. The ratio $|\alpha / \beta|$ is ranging between approximately 1.5 to 8 for these twodimensional systems with electron densities of the order of $\sim 10^{11}-10^{12} \mathrm{~cm}^{-2}$.

The semiclassical Boltzmann theory is applicable when the following two conditions are satisfied. First, the particle's de Broglie wavelength must be smaller than the mean-free path. At low temperatures (as compared to the Fermi temperature) the condition implies that

$$
n_{e}>\frac{m}{\hbar \tau}
$$

where $\tau=m \mu / e$. For the above InAs and GaAs twodimensional systems ${ }^{42} \mathrm{~m} /(\hbar \tau)$ is of the order of $10^{10} \mathrm{~cm}^{-2}$ and $10^{9} \mathrm{~cm}^{-2}$, respectively, so the inequality (24) can be safely met.

The second condition requires that the smearing of the spin-split bands due to disorder is smaller than the spin splitting energy $E_{+, \mathbf{k}}-E_{-, \mathbf{k}}$. Since the AMR we study is due to the SOI in the band structure (rather than in the scatterers) it remains nonzero only in the strong SOI/weak disorder regime. As a consequence, the concentration must also fulfill the following inequality

$$
n_{e}>\hbar^{2} / 8 \pi \kappa_{\theta}^{2} \tau^{2}
$$

Assuming a pure Rashba system (i.e., $\kappa_{\theta} \equiv \alpha$ ), the right-hand side in Eq. (25) is of the order of $10^{9} \mathrm{~cm}^{-2}$ for both InAs and $\mathrm{GaAs}$, respectively, so the condition is again satisfied for typical electron densities. Introducing magnetic impurities will certainly decrease the mobility of the two-dimensional systems, nevertheless, conditions (24) and (25) might remain satisfied for feasible electron densities. We also note that the inequality (25) can be reformulated in terms of the mean free path $l_{\tau}$ and spin precession length, which can be roughly estimated as $\lambda_{s} \sim \hbar^{2} /(m \alpha)$. Namely, $l_{\tau}$ must be larger than $\lambda_{s}$ so that an electron randomizes its spin orientation due to the spin-orbit precession between two subsequent scattering events. This restriction corresponds to the approximation which neglects the off-diagonal elements of the nonequilibrium distribution function in the spin space.

Having established parameter range of the validity of the Boltzmann approach we can now return to points (i)-(iii) from the beginning of this section and comment on the expected AMRs for realistic material parameters. Since for short-range impurities the AMR is weak when $|\alpha| \approx|\beta|$ let us assume pure Rashba model only. By a direct inspection of the results in Tables I and III we find that the ratio between the isotropic and anisotropic part, $\sigma_{0}$ and $\sigma_{1}$, of the conductivity tensor depends on the SOI strength and electron density and can be estimated as

$$
\frac{\sigma_{1}}{\sigma_{0}} \sim \frac{1}{\pi n_{e}}\left(\frac{m \alpha}{\hbar^{2}}\right)^{2} .
$$

For usual electron densities $\sim 10^{11} \mathrm{~cm}^{-2}$, this ratio will be of the order of 0.01 for a pure magnetic impurity potential, implying weak AMR of the order of $1 \%$. By depleting the minority band, the ratio $\sigma_{1} / \sigma_{0}$ can be enhanced and the AMR can reach up to $100 \%$ (recall Fig. 5). However, corresponding densities of $n_{e} \approx 10^{9} \mathrm{~cm}^{-2}$ are relatively low compared to densities of typical experimental two-dimensional electron systems and also we then move towards the edge of the validity of the Boltzmann theory.

The AMR will be further reduced by the presence of another impurities than the (electro-)magnetic ones. In terms of resistivities, this follows from the Matthiessen's rule stating that the total resistivity is a sum of resistivities due to the particular scattering mechanisms. ${ }^{43}$ Since scattering from pure nonmagnetic impurities yields zero contribution to the type of AMR discussed in this paper the overall relative magnetic anisotropy of the resistivity is suppressed by their presence.

On the other hand, for impurities containing combined electromagnetic potentials which add up coherently during the scattering, the AMR is expected to be largely enhanced even in the high density regime. The strongest AMR is predicted for similar strength of the magnetic and electric parts of the scattering potential. This applies, e.g., to $\mathrm{Mn}$ in GaAs which acts both as a charged dopant and a localized magnetic impurity, and the AMRs in GaAs:Mn can reach $\sim 10 \% .{ }^{44}$ In the present paper, we however wish to limit our investigation of models beyond the Rashba-Dresselhaus one to the qualitative discussion of Sec. II D complemented by the exact Boltzmann equation AMR given in Appendix B. We refer the reader to Refs. 29, 37, and 38 for a more quantitative discussion of AMR in (Ga, Mn)As and finally remark that the realization of large AMRs in Rashba-Dresselhaus systems with electromagnetic impurities will require doping with magnetic donors. 


\section{ACKNOWLEDGMENTS}

The work was funded through Præmium Academiæ and Contracts No. AV0Z10100521, No. LC510, No. KAN400100652, and No. FON/06/E002 of GA ČR, and Grant No. KJB100100802 of GA AV of the Czech republic, by the NAMASTE (FP7 Grant No. 214499) and SemiSpinNet projects (FP7 Grant No. 215368), by DARPA, and by DFG via Grant No. SFB 689. One of us (K.V.) gratefully acknowledges inspiring discussions with Vladimir I. Fal'ko on the exact solution of the Boltzmann equation, hospitality of Roland Winkler at the ANL, and helpful assistance with programming of Lukáš Kripner in the adventurous period of work on Appendix B.

\section{APPENDIX A: BOLTZMANN EQUATION FOR RASHBA- DRESSELHAUS HAMILTONIAN}

In order to determine the nonequilibrium distribution function, we insert the ansatz (22) into the Boltzmann equation (8) and obtain a set of four linear equations for parameters $a_{x, y}^{\hat{e}_{M}}, b_{x, y}^{\hat{e}_{M}}$, one for each direction of electric field $(x, y)$ and each magnetization direction $\hat{e}_{M}$. For the scatterers magnetized along $\hat{x}(\hat{y})$ axis and $\mathbf{E}=\left(E_{x}, 0\right)$, we get

$$
\begin{aligned}
a_{x}= & \mp \frac{\beta^{2}-\alpha^{2}}{2} \\
& \pm \frac{1}{2}\left[a_{x} \frac{\beta^{2}-\alpha^{2}}{\left|\alpha^{2}-\beta^{2}\right|}+b_{x} \frac{\beta^{2}-\alpha^{2}}{2 \alpha \beta}\left(1-\frac{\alpha^{2}+\beta^{2}}{\left|\alpha^{2}-\beta^{2}\right|}\right)\right], \\
b_{x}= & \mp \frac{\left(\alpha^{2}+\beta^{2}\right)^{2}}{4 \alpha \beta}\left(\frac{\left|\alpha^{2}-\beta^{2}\right|}{\alpha^{2}+\beta^{2}}-1\right) \\
& \pm \frac{1}{2}\left[a_{x} \frac{\alpha^{2}+\beta^{2}}{2 \alpha \beta}\left(1-\frac{\alpha^{2}+\beta^{2}}{\left|\alpha^{2}-\beta^{2}\right|}\right)+b_{x} \frac{\alpha^{2}+\beta^{2}}{\left|\alpha^{2}-\beta^{2}\right|}\right] .
\end{aligned}
$$

The choice $\mathbf{E}=\left(0, E_{y}\right)$ leads to

$$
\begin{aligned}
a_{y}= & \pm \frac{\alpha^{4}-\beta^{4}}{4 \alpha \beta}\left(\frac{\left|\alpha^{2}-\beta^{2}\right|}{\alpha^{2}+\beta^{2}}-1\right) \\
& \pm \frac{1}{2}\left[a_{y} \frac{\beta^{2}-\alpha^{2}}{\left|\alpha^{2}-\beta^{2}\right|}+b_{y} \frac{\beta^{2}-\alpha^{2}}{2 \alpha \beta}\left(1-\frac{\alpha^{2}+\beta^{2}}{\left|\alpha^{2}-\beta^{2}\right|}\right)\right] \\
b_{y}= & \mp \frac{\alpha^{2}+\beta^{2}}{2} \\
& \pm \frac{1}{2}\left[b_{y} \frac{\alpha^{2}+\beta^{2}}{\left|\alpha^{2}-\beta^{2}\right|}+a_{y} \frac{\alpha^{2}+\beta^{2}}{2 \alpha \beta}\left(1-\frac{\alpha^{2}+\beta^{2}}{\left|\alpha^{2}-\beta^{2}\right|}\right)\right] .
\end{aligned}
$$

Here, we skip the $\hat{e}_{M}$ superscript for brevity and relate the upper and lower signs to the magnetization $\mathbf{M}$ along $\hat{x}$ and $\hat{y}$ axes, respectively.

For scatterers magnetized along the [110] axis we have

$$
\begin{aligned}
& a_{x} \frac{\alpha^{2}+\beta^{2}-\left|\alpha^{2}-\beta^{2}\right|}{4 \alpha \beta}+\frac{b_{x}}{2}+a_{x} \\
& =\alpha \beta\left[1+\frac{\left(\alpha^{2}+\beta^{2}\right)^{2}}{4 \alpha^{2} \beta^{2}}\left(\frac{\left|\alpha^{2}-\beta^{2}\right|}{\alpha^{2}+\beta^{2}}-1\right)\right], \\
& b_{x} \frac{\alpha^{2}+\beta^{2}-\left|\alpha^{2}-\beta^{2}\right|}{4 \alpha \beta}+b_{x}+\frac{a_{x}}{2}=\frac{\left|\alpha^{2}-\beta^{2}\right|}{2},
\end{aligned}
$$

while equations for $a_{y}$ and $b_{y}$ can be obtained from Eqs. (A5) and (A6) by the substitution $a_{x} \rightarrow b_{y}, b_{x} \rightarrow a_{y}$.

\section{Impurity magnetization along the [100]-axis}

Here, we assume that impurities are magnetized along the $x$ axis, i.e., the scattering potential is proportional to $\sigma_{x}$. If $\alpha>\beta$ then solution of Eqs. (A1) and (A2) and Eqs. (A3) and (A4) with upper sign reads

$$
a_{x}=\frac{\alpha^{4}-\beta^{4}}{3 \alpha^{2}+\beta^{2}}, \quad b_{x}=\frac{8 \alpha^{3} \beta}{3 \alpha^{2}+\beta^{2}}-2 \alpha \beta,
$$

and

$$
a_{y}=2 \alpha \beta-\frac{8 \alpha^{3} \beta}{3 \alpha^{2}+\beta^{2}}, \quad b_{y}=-\frac{24 \alpha^{4}}{3 \alpha^{2}+\beta^{2}}+7 \alpha^{2}-\beta^{2} .
$$

In the opposite case $\beta>\alpha$ the coefficients are

$$
\begin{gathered}
a_{x}=-\frac{\alpha^{4}-4 \beta^{2} \alpha^{2}+3 \beta^{4}}{\alpha^{2}+3 \beta^{2}}, \quad b_{x}=\frac{2 \alpha\left(\alpha^{2}-\beta^{2}\right) \beta}{\alpha^{2}+3 \beta^{2}}, \\
a_{y}=-\frac{2 \alpha\left(\alpha^{2}-\beta^{2}\right) \beta}{\alpha^{2}+3 \beta^{2}}, \quad b_{y}=\frac{\beta^{4}-\alpha^{4}}{\alpha^{2}+3 \beta^{2}} .
\end{gathered}
$$

\section{Impurity magnetization along the [010] axis}

The scattering potential is proportional to $\sigma_{y}$ in this case. If $\alpha>\beta$ then the solution of Eqs. (A1) and (A2) and Eqs. (A3) and (A4) with lower signs is given by

$$
\begin{gathered}
a_{x}=-\frac{24 \alpha^{4}}{3 \alpha^{2}+\beta^{2}}+7 \alpha^{2}-\beta^{2}, \quad b_{x}=2 \alpha \beta-\frac{8 \alpha^{3} \beta}{3 \alpha^{2}+\beta^{2}}, \\
a_{y}=\frac{8 \alpha^{3} \beta}{3 \alpha^{2}+\beta^{2}}-2 \alpha \beta, \quad b_{y}=\frac{\alpha^{4}-\beta^{4}}{3 \alpha^{2}+\beta^{2}},
\end{gathered}
$$

while in the opposite case $(\beta>\alpha)$, the coefficients read

$$
\begin{gathered}
a_{x}=\frac{\beta^{4}-\alpha^{4}}{\alpha^{2}+3 \beta^{2}}, \quad b_{x}=-\frac{2 \alpha\left(\alpha^{2}-\beta^{2}\right) \beta}{\alpha^{2}+3 \beta^{2}}, \\
a_{y}=\frac{2 \alpha\left(\alpha^{2}-\beta^{2}\right) \beta}{\alpha^{2}+3 \beta^{2}}, \quad b_{y}=-\frac{\alpha^{4}-4 \beta^{2} \alpha^{2}+3 \beta^{4}}{\alpha^{2}+3 \beta^{2}} .
\end{gathered}
$$

\section{Impurity magnetization along the [110] axis}

Here, the scattering potential is proportional to $\frac{1}{2}\left(\sigma_{x}+\sigma_{y}\right)$. If $\alpha>\beta$ then the coefficients $a_{x, y}$ and $b_{x, y}$ read 


$$
\begin{aligned}
& a_{x}=-\frac{\alpha^{3}-3 \beta \alpha^{2}+\beta^{2} \alpha+\beta^{3}}{3 \alpha+\beta}, \quad b_{x}=\frac{2 \alpha^{2}(\alpha-\beta)}{3 \alpha+\beta}, \\
& a_{y}=\frac{2 \alpha^{2}(\alpha-\beta)}{3 \alpha+\beta}, \quad b_{y}=-\frac{\alpha^{3}-3 \beta \alpha^{2}+\beta^{2} \alpha+\beta^{3}}{3 \alpha+\beta} .
\end{aligned}
$$

In the opposite case $\beta>\alpha$ we have

$$
\begin{aligned}
& a_{x}=-\frac{\alpha^{3}+\beta \alpha^{2}-3 \beta^{2} \alpha+\beta^{3}}{\alpha+3 \beta}, \quad b_{x}=\frac{2 \beta^{2}(\beta-\alpha)}{\alpha+3 \beta}, \\
& a_{y}=\frac{2 \beta^{2}(\beta-\alpha)}{\alpha+3 \beta}, \quad b_{y}=-\frac{\alpha^{3}+\beta \alpha^{2}-3 \beta^{2} \alpha+\beta^{3}}{\alpha+3 \beta} .
\end{aligned}
$$

The case when the impurities are magnetized along the [1 $1 \overline{1} 0]$ axis can be treated in the same way.

To write down the conductivity for arbitrary $\alpha$ and $\beta$ it is convenient to define

$$
\sigma_{0}=\frac{e^{2} \tau}{m} n_{e}
$$

where the electron concentration at $E_{F} \geq 0$ can be exactly expressed as

$$
n_{e}=\frac{m E_{F}}{\pi \hbar^{2}}+\left(\frac{m}{\hbar^{2}}\right)^{2} \frac{\alpha^{2}+\beta^{2}}{\pi} .
$$

Thus defined $\sigma_{0}$ becomes identical with the Drude formula when $\alpha=\beta=0$. In fact, $\sigma_{0}$ times unity $2 \times 2$ matrix describes the conductivity of a 2DEG due to the nonmagnetic shortrange scatterers, see Ref. 14. In the presence of magnetized scatterers the conductivity acquires an additional term $\widehat{\sigma}_{1}$ which is summarized in Table III. To obtain the total conductivity tensor one has to sum up both these terms, i.e., $\hat{\sigma}$ $=1 \sigma_{0}+\widehat{\sigma_{1}}$. Conductivity tensors under special conditions in Table I can be recovered by a proper choice of $\alpha, \beta$ in Table III. Table III thus summarizes the main computational results of this paper. They describe an additional term in the electrical conductivity of a 2DEG confined in a [001]-grown III-V semiconductor heterostructure due to the magnetized elastic scatterers.

\section{APPENDIX B: BOLTZMANN EQUATION FOR KOHN- LUTTINGER HAMILTONIAN}

Nontrivial exact analytical solutions to the Boltzmann equation (8) exist also for some models in $d=3$ dimensions. The one described in Sec. II D constitutes one such example and we outline here the main steps needed to calculate the AMR in this model and thus confirm the appropriateness of the sketch on Fig. 4(b).

Physically, the model concerns carriers of the two heavyhole $\Gamma_{8}$ bands (HH bands) scattered off magnetic impurities. The band structure can formally be viewed as the $\left(\gamma_{1}\right.$ $\left.-2 \gamma_{2}\right) /\left(\gamma_{1}+2 \gamma_{2}\right) \rightarrow 0$ limit (negligible light-hole density of states) of the Hamiltonian (6) with $j_{x, y, z}=3 s_{x, y, z}$ while scatterers uniformly polarized along $z$ direction are modeled by $V$ $\propto s_{z}$ in terms of Eq. (9). Explicit expressions for the spin matrices $s_{x, y, z}$ can be found, e.g., in the Appendix of Ref. 45 . Without going into details, we remark that this model could be used to describe the AMR in (metallic) $p$-type III-V or II-VI semiconductors with dilute $\mathrm{Mn}$ impurities if their charge is either zero or strongly screened; ${ }^{29,45,47} \mathrm{Mn}$ atom $d$-states hybridize with the host valence band and create ${ }^{46}$ a $\vec{k}$-independent ${ }^{12}$ impurity potential $V \propto \hat{e}_{M} \cdot \mathbf{s}$. Relevant values of the proportionality constant and host material band structure parameters $\gamma_{1}, \gamma_{2}$ can be found in Ref. 47 . We also stress that we will be treating a model where the densities of states of the two involved $(\mathrm{HH})$ bands are equal $(h \rightarrow 0)$ and this is of course (again) only an approximation to realistic systems.

Nonequilibrium distributions due to applied electric field turn out to be the same for both HH bands in such a model, and we are required to solve three decoupled integral equations

$$
\begin{gathered}
\sqrt{4 \pi / 3} \tau Y_{1}^{0}(\Omega)=\bar{w}(\Omega) c(\Omega)-\int d \Omega^{\prime} w\left(\Omega, \Omega^{\prime}\right) c\left(\Omega^{\prime}\right), \\
-\sqrt{8 \pi / 3} \tau Y_{1}^{1}(\Omega)=\bar{w}(\Omega) p(\Omega)-\int d \Omega^{\prime} w\left(\Omega, \Omega^{\prime}\right) p\left(\Omega^{\prime}\right), \\
\sqrt{8 \pi / 3} \tau Y_{1}^{-1}(\Omega)=\bar{w}(\Omega) q(\Omega)-\int d \Omega^{\prime} w\left(\Omega, \Omega^{\prime}\right) q\left(\Omega^{\prime}\right),
\end{gathered}
$$

where $\tau$ is defined by the three-dimensional (3D) analogy of Eq. (11), $\Omega$ denotes a compound variable $\varphi, \vartheta$ parameterizing the unit sphere, so that $\int d \Omega=\int_{0}^{\pi} \sin \vartheta d \vartheta \int_{0}^{2 \pi} d \varphi$,

$$
\begin{aligned}
& w\left(\Omega, \Omega^{\prime}\right)=-\frac{2 \pi}{15}\left(Y_{2}^{-1} Y_{2}^{1^{\prime}}+Y_{2}^{1} Y_{2}^{-1^{\prime}}\right)+\frac{10 \pi}{9} Y_{0}^{0} Y_{0}^{0^{\prime}} \\
&+\frac{8 \pi}{9 \sqrt{5}}\left(Y_{0}^{0} Y_{2}^{0^{\prime}}+Y_{2}^{0} Y_{0}^{0^{\prime}}\right)+\frac{2 \pi}{9} Y_{2}^{0} Y_{2}^{0^{\prime}} \\
&-\frac{2 \pi}{15}\left(Y_{2}^{-2} Y_{2}^{2^{\prime}}+Y_{2}^{2} Y_{2}^{-2^{\prime}}\right), \\
& \bar{w}(\Omega)=\int d \Omega^{\prime} w\left(\Omega, \Omega^{\prime}\right)=\frac{2 \pi \sqrt{4 \pi}}{3}\left(\frac{4}{3 \sqrt{5}} Y_{2}^{0}+\frac{5}{3} Y_{0}^{0}\right),
\end{aligned}
$$

and $Y_{l}^{m}=Y_{l}^{m}(\Omega), Y_{l}^{m^{\prime}}=Y_{l}^{m}\left(\Omega^{\prime}\right)$ denote spherical harmonics normalized to $\int d \Omega Y_{l}^{m, *}(\Omega) Y_{l^{\prime}}^{m^{\prime}}(\Omega)=\delta_{l l^{\prime}} \delta_{m m^{\prime}}$ according to the Condon-Shortley convention.

Nonequilibrium distribution under the effect of $\mathbf{E}$ $=\left(E_{x}, E_{y}, E_{z}\right)$ is then

$$
\begin{aligned}
f(\mathbf{k})-f^{0}\left(E_{k}\right)= & e v \frac{\partial f^{0}\left(E_{k}\right)}{\partial E_{k}}\left\{\frac{1}{2}[p(\Omega)+q(\Omega)] E_{x}\right. \\
& \left.-\frac{1}{2} i[p(\Omega)-q(\Omega)] E_{y}+c(\Omega) E_{z}\right\}
\end{aligned}
$$

for the both bands (which are in the $h \rightarrow 0$ approximation identical), and conductivities implied in the spirit of Eq. (12) are 


$$
\begin{gathered}
\sigma_{x x}=\int d \Omega \frac{1}{8}[p(\Omega)+q(\Omega)]\left[Y_{1}^{-1}(\Omega)-Y_{1}^{1}(\Omega)\right], \\
\sigma_{y y}=\int d \Omega \frac{1}{8}[p(\Omega)-q(\Omega)]\left[Y_{1}^{1}(\Omega)+Y_{1}^{-1}(\Omega)\right], \\
\sigma_{z z}=\int d \Omega \frac{\sqrt{2}}{4} c(\Omega) Y_{1}^{0}(\Omega) .
\end{gathered}
$$

in units of $\sqrt{3 / 2 \pi} \cdot e^{2} n_{e} / m$. Using analytical solutions of Eqs. (B1) corresponding to scattering amplitudes (B2), we find

$$
\mathrm{AMR}=2 \frac{-6+5 \sqrt{2} \arctan \sqrt{2}}{2+\sqrt{2} \arctan \sqrt{2}} \approx 0.45,
$$

according to our definition of AMR (1), that is resistance parallel to the magnetization is higher. It is thus confirmed that sketches for pure magnetic scattering in Fig. 4(b) appropriately describe conductivity calculated by exactly solving the Boltzmann equation. We also obtained the same result (B4) within the Keldysh formalism ${ }^{21,23}$ where conductivity $\sigma_{x x}$ turns out to be proportional to $\left\langle v_{x} \delta v_{x} \delta\right\rangle_{t}$ with $v_{x}$ $=\partial H_{K L} / \partial k_{x}, \quad \delta=G^{R}-G^{A}, G^{A}=\left(G^{R}\right)^{\dagger}$ and $\left(G^{R}\right)^{-1}=E_{F}-H_{K L}$ $-\Sigma^{R}$ with self-energy $\Sigma^{R} \propto s_{z}^{2}$. Brackets $\langle\ldots\rangle_{t}$ in this Kuboformula-type result ${ }^{48}$ mean trace in the space of $4 \times 4$ matrices and integration over the $k$ space. Conductivity $\sigma_{z z}$ is analogous $\left(v_{x}\right.$ is replaced by $v_{z}, \Sigma^{R}$ stays unchanged).

We finally remark that Eqs. (B1) are completely analogous to the two equations [Eqs. (8) and (7)] of the 2D case in Ref. 32. Solution of those equations was constructed in the form of a Fourier series or a modified Fourier series as explained in the note added in proof of that reference. In our current 3D problem defined by Eqs. (B1) and (B2), if expanded in terms of modified spherical harmonics $Y_{l}^{m}(\Omega) / \bar{w}(\Omega)$, the solutions $c(\Omega), p(\Omega), q(\Omega)$ are found to contain only few terms [the harmonics present in the lefthand-sides of Eqs. (B1); note the analogy to the discussion of Sec. III A and Eq. (22) when spherical harmonics replace sines and cosines]. During the calculations we have to be however cautious as the integrals of Eqs. (B1) do not contain simple scalar products of spherical harmonics where orthogonality relations apply.
${ }^{1}$ L. Chien and C. R. Westgate, The Hall Effect and Its Applications (Plenum, New York, 1980).

${ }^{2}$ J. Sinova, T. Jungwirth, and J. Černe, Int. J. Mod. Phys. B 18 , 1083 (2004).

${ }^{3}$ N. A. Sinitsyn, J. Phys.: Cond. Matt. 20, 023201 (2008).

${ }^{4}$ T. Jungwirth, Q. Niu, and A. H. MacDonald, Phys. Rev. Lett. 88, 207208 (2002).

${ }^{5}$ T. Dietl, F. Matsukura, H. Ohno, J. Cibert, and D. Ferrand, in Recent Trends in Theory of Physical Phenomena in High Magnetic Fields, edited by I. Vagner (Kluwer, Dordrecht, 2003), p. 197.

${ }^{6}$ D. Ruzmetov, J. Scherschligt, D. V. Baxter, T. Wojtowicz, X. Liu, Y. Sasaki, J. K. Furdyna, K. M. Yu, and W. Walukiewicz, Phys. Rev. B 69, 155207 (2004).

${ }^{7}$ S. H. Chun, Y. S. Kim, H. K. Choi, I. T. Jeong, W. O. Lee, K. S. Suh, Y. S. Oh, K. H. Kim, Z. G. Khim, J. C. Woo, and Y. Park, Phys. Rev. Lett. 98, 026601 (2007).

${ }^{8}$ G. Mihály, M. Csontos, S. Bordács, I. Kézsmárki, T. Wojtowicz, X. Liu, B. Jankó, and J. K. Furdyna, Phys. Rev. Lett. 100, 107201 (2008).

${ }^{9}$ L.-F. Arsenault, B. Movaghar, P. Desjardins, and A. Yelon, Phys. Rev. B 77, 115211 (2008).

${ }^{10}$ Y. Pu, D. Chiba, F. Matsukura, H. Ohno, and J. Shi, Phys. Rev. Lett. 101, 117208 (2008).

${ }^{11}$ F. Matsukura, H. Ohno, and T. Dietl, in Handbook of Magnetic Materials, edited by K. H. J. Buschow (Elsevier, Amsterdam, 2002), Vol. 14, p. 1, From Ohno Lab Homepage.

${ }^{12}$ T. Jungwirth, J. Sinova, J. Mašek, J. Kučera, and A. H. MacDonald, Rev. Mod. Phys. 78, 809 (2006).

${ }^{13}$ V. K. Dugaev, P. Bruno, M. Taillefumier, B. Canals, and C. Lacroix, Phys. Rev. B 71, 224423 (2005).

${ }^{14}$ M. Trushin and J. Schliemann, Phys. Rev. B 75, 155323 (2007).

${ }^{15}$ N. A. Sinitsyn, A. H. MacDonald, T. Jungwirth, V. K. Dugaev, and J. Sinova, Phys. Rev. B 75, 045315 (2007).

${ }^{16}$ J. I. Inoue, T. Kato, Y. Ishikawa, H. Itoh, G. E. W. Bauer, and L. W. Molenkamp, Phys. Rev. Lett. 97, 046604 (2006).

${ }^{17}$ S. Onoda, N. Sugimoto, and N. Nagaosa, Phys. Rev. B 77, 165103 (2008)

${ }^{18}$ T. S. Nunner, N. A. Sinitsyn, M. F. Borunda, V. K. Dugaev, A. A. Kovalev, Ar. Abanov, C. Timm, T. Jungwirth, J. I. Inoue, A. H. MacDonald, and J. Sinova, Phys. Rev. B 76, 235312 (2007).

${ }^{19}$ T. S. Nunner, G. Zaránd, and F. von Oppen, Phys. Rev. Lett. 100, 236602 (2008).

${ }^{20}$ M. F. Borunda, T. S. Nunner, T. Luck, N. A. Sinitsyn, C. Timm, J. Wunderlich, T. Jungwirth, A. H. MacDonald, and J. Sinova, Phys. Rev. Lett. 99, 066604 (2007).

${ }^{21}$ A. A. Kovalev, K. Výborný, and J. Sinova, Phys. Rev. B 78, 041305(R) (2008).

${ }^{22}$ J. Sinova and A. H. MacDonald, in Spintronics, edited by T. D. D. D. A. M. K. H. Ohno (Elsevier, New York, 2008), Vol. 82 of Semicond. Semimet., p. 45.

${ }^{23}$ A. A. Kovalev, Y. Tserkovnyak, K. Vyborny, and J. Sinova, Phys. Rev. B 79, 195129 (2009).

${ }^{24}$ J. Smit, Physica 17, 612 (1951).

${ }^{25}$ O. Jaoul, I. A. Campbell, and A. Fert, J. Magn. Magn. Mater. 5, 23 (1977)

${ }^{26}$ T. McGuire and R. Potter, IEEE Trans. Magn. 11, 1018 (1975).

${ }^{27}$ J. Banhart and H. Ebert, Europhys. Lett. 32, 517 (1995).

${ }^{28}$ S. Khmelevskyi, K. Palotás, L. Szunyogh, and P. Weinberger, Phys. Rev. B 68, 012402 (2003).

${ }^{29}$ A. W. Rushforth, K. Výborný, C. S. King, K. W. Edmonds, R. P. Campion, C. T. Foxon, J. Wunderlich, A. C. Irvine, P. Vašek, V. Novák, K. Olejnik, J. Sinova, T. Jungwirth, and B. Gallagher, Phys. Rev. Lett. 99, 147207 (2007).

${ }^{30}$ T. Jungwirth, B. L. Gallagher, and J. Wunderlich, in Spintroncs, edited by Tomasz Dietl, David D. Awschalom, Maria Kaminska, 
and Hideo Ohno (Elsevier, New York, 2008), Vol. 82 of Semicond. Semimet., p. 135.

${ }^{31}$ T. Jungwirth, M. Abolfath, J. Sinova, J. Kučera, and A. H. MacDonald, Appl. Phys. Lett. 81, 4029 (2002).

${ }^{32}$ K. Výborný, A. A. Kovalev, J. Sinova, and T. Jungwirth, Phys. Rev. B 79, 045427 (2009).

${ }^{33}$ J. Schliemann, J. C. Egues, and D. Loss, Phys. Rev. Lett. 90, 146801 (2003).

${ }^{34}$ J. Schliemann and D. Loss, Phys. Rev. B 68, 165311 (2003).

${ }^{35}$ B. A. Bernevig, J. Orenstein, and S.-C. Zhang, Phys. Rev. Lett. 97, 236601 (2006).

${ }^{36}$ I. Vurgaftman, J. R. Meyer, and L. R. Ram-Mohan, J. Appl. Phys. 89, 5815 (2001).

${ }^{37}$ A. W. Rushforth, K. Výborný, C. S. King, K. W. Edmonds, R. P. Campion, C. T. Foxon, J. Wunderlich, A. C. Irvine, V. Novák, K. Olejník, A. Kovalev, J. Sinova, T. Jungwirth, and B. Gallagher, J. Mag. Magn. Mater. 321, 1001 (2009).

${ }^{38}$ K. Vyborny, J. Kucera, J. Sinova, A. W. Rushforth, B. L. Gallagher, and T. Jungwirth, arXiv:0906.3151, Phys. Rev. B (to be published).

${ }^{39}$ J. Luo, H. Munekata, F. F. Fang, and P. J. Stiles, Phys. Rev. B 41, 7685 (1990).

${ }^{40}$ Y. B. Vasilyev, S. D. Suchalkin, S. V. Ivanov, B. Y. Meltser, and P. S. Kop'ev, Phys. Status Solidi B 240, R8 (2003).

${ }^{41}$ S. D. Ganichev, V. V. Bel'kov, L. E. Golub, E. L. Ivchenko, P. Schneider, S. Giglberger, J. Eroms, J. D. Boeck, G. Borghs, W. Wegscheider et al., Phys. Rev. Lett. 92, 256601 (2004).

${ }^{42}$ S. Giglberger, L. E. Golub, V. V. Bel'kov, S. N. Danilov, D. Schuh, C. Gerl, F. Rohlfing, J. Stahl, W. Wegscheider, D. Weiss et al., Phys. Rev. B 75, 035327 (2007).

${ }^{43}$ Matthiessen's rule can, however, be only applied on a qualitative level in anisotropic systems. See for example J. S. Dugdale and
Z. S. Basinski, Phys. Rev. 157, 552 (1967).

${ }^{44}$ T. Jungwirth, J. Sinova, K. Y. Wang, K. W. Edmonds, R. P. Campion, B. L. Gallagher, C. T. Foxon, Q. Niu, and A. H. MacDonald, Appl. Phys. Lett. 83, 320 (2003).

${ }^{45}$ M. Abolfath, T. Jungwirth, J. Brum, and A. H. MacDonald, Phys. Rev. B 63, 054418 (2001).

${ }^{46}$ J. R. Schrieffer and P. A. Wolff, Phys. Rev. 149, 491 (1966).

${ }^{47}$ T. Dietl, H. Ohno, and F. Matsukura, Phys. Rev. B 63, 195205 (2001). To complete the link between this reference and Eq. (9), we note that $\beta N_{0}$ shown in Appendix $\mathrm{C}$ of Dietl, Ohno, and Matsukura equals in our notation to $J_{p d} n / x$ in a $\left(\mathrm{III}_{1-x}, \mathrm{Mn}_{x}\right) \mathrm{V}$ material and the scattering potential including the proportionality constant reads $V=J_{p d} S_{\mathrm{Mn}} \hat{e}_{M} \cdot \mathbf{s}$ where $S_{\mathrm{Mn}}=\frac{5}{2}$ is the total spin of involved $\mathrm{Mn} d$ electrons and $\mathbf{s}=\left(s_{x}, s_{y}, s_{z}\right)$. The proportionality constant drops out in the expression for the AMR but it still must provide for the Mn impurities to be the dominant source of scattering. Note that the proportionality constant is also closely related to $h$ in Eq. (6).

${ }^{48}$ See, e.g., Eq. (11) in P. Středa and L. Smrčka, Phys. Status Solidi B 70, 537 (1975), which is a more general result (finite temperature, more general form of disorder, magnetic field).

${ }^{49}$ This combined effect of SOI and carrier polarization is the third type of microscopic mechanism that can lead to AMR. In $(\mathrm{Ga}, \mathrm{Mn}) \mathrm{As}$, however, carrier-polarization-related anisotropy in group velocities is also weak, see Refs. 29, 37, and 38.

${ }^{50}$ The situation when two distinct types of impurities are present is treated in Ref. 31 and also discussed in Ref. 38.

${ }^{51}$ See Eq. (15) and Table II for explicit expressions in the case of $V \propto \hat{e}_{M} \cdot \boldsymbol{\sigma}$. For $V \propto 1$, we find $\Sigma_{i^{\prime}} w\left(i, \theta ; i^{\prime}, \theta\right)$ independent of $\theta, \theta^{\prime}$ using $w\left(i, \theta ; i^{\prime}, \theta^{\prime}\right)=1+i i^{\prime} \cos \left(\theta-\theta^{\prime}\right)$.

${ }^{52}$ See also discussion of physical relevance of this singularity in Sec. IV or Ref. 32. 